\title{
FLOWS OF DEHAVEN FLUID IN SYMMETRICALLY CURVED CAPILLARY FISSURES AND TUBES
}

\author{
A. WALICKA*, J.FALICKI and P.JURCZAK \\ University of Zielona Góra, Faculty of Mechanical Engineering \\ ul. Szafrana 4, 65-516 Zielona Góra, POLAND \\ E-mail: A.Walicka@ijame.uz.zgora.pl
}

\begin{abstract}
In this paper, an analytical method for deriving the relationships between the pressure drop and the volumetric flow rate in laminar flow regimes of DeHaven type fluids through symmetrically corrugated capillary fissures and tubes is presented. This method, which is general with regard to fluid and capillary shape, can also be used as a foundation for different fluids, fissures and tubes. It can also be a good base for numerical integration when analytical expressions are hard to obtain due to mathematical complexities.

Five converging-diverging or diverging-converging geometrics, viz. variable cross-section, parabolic, hyperbolic, hyperbolic cosine and cosine curve, are used as examples to illustrate the application of this method. Each example is concluded with a presentation of the formulae for the velocity flow on the outer surface of a thin porous layer. Upon introduction of hindrance factors, these formulae may be presented in the most general forms.
\end{abstract}

Key words: DeHaven fluid, laminar flow, symmetrically corrugated capillaries, capillary fissures or tubes.

\section{Introduction}

Fluid flows and transport phenomena through the classical "ground" or "soil" are encountered literally everywhere in everyday life, in nature (ground water), industries (composite materials, building materials, etc.) as well as in biosystems (aquifer ecosystems, human organs, etc.) and other domains such as e.g., membranes used in biofuel cell applications.

The reason is that except metals, some plastics and dense rocks, almost all solid and semisolid materials can be considered as "porous" in varying degrees. Hence, there exist many types of different technologies that depend on theories used to describe transport phenomena in porous media.

There are many practical applications that can be modelled or approximated as transport through porous media. These applications have been discussed by Bear [1], Greenkorn [2], Nield and Bejan [3], Vafai [4-7], Hadim and Vafai [8], Vafai and Hadim [9].

In the works cited above, the porous medium is viewed as a continuum with solid and fluid phases in thermal equilibrium, isotropic, homogeneous and saturated with an incompressible Newtonian fluid. Vafai and Tien [10] presented a comprehensive analysis of the generalized transport through porous media and developed a set of governing equations utilizing the local VAT (volume averaging theory/technique) or/and the REV (representative elementary volume) technique. The final forms of these equations can be found in the works by Amiri and Vafai [11], Alazmi and Vafai [12], Khanafer et al. [13]. Peng and Wu [14] describe a series of different experimental observations and associated theoretical investigations conducted to understand the transport phenomena with or without phase change and chemical reaction and concerning a wide range of practical applications. Fault and fracture zones are often highly-complex heterogeneities that can have a significant effect on the fluid flow within petroleum reservoirs on length scales of less than $1 \mu \mathrm{m}$ to more than $10 \mathrm{~km}$. It is therefore important to incorporate their properties in developing simulation models. Harris et al. [15] describe some of the numerical techniques being used to model the effects of faults and fractures on fluid flow. Other theoretical models are groundwater models (Karamouz et al. [16], Yeh [17]) which have been used extensively for groundwater flow analysis, pollution transport and groundwater management.

\footnotetext{
* To whom correspondence should be addressed
} 
Another way to study the flows in porous media is to use conceptual models; a great example of such models are PNMs (pore network models). These models have gained a lot of popularity among researchers since they are much more systematic than the real pore space of a soil and have been used in a variety of fields such as petroleum engineering, hydrology and soil physics. In these models, the soil pore space is modelled by a discrete network of pores that are connected by throats (Jivkov et al. [18]). Throats in PNMs may be prismatic or nonprismatic, mainly converging-diverging types (Xiong et al. [19]). The studies of the Newtonian flow in circular prismatic tubes (otherwise speaking: circular tubes of constant cross-sections) were performed by Mazaheri et al. [20], Joekar-Niaser et al. [21] and Nsir and Schafer [22]. The flow in non-prismatic tubes, namely in conical tubes was studied by Held and Celia [23], Hilpert et al. [24] and by Acharya et al. [25].

The studies of non-Newtonian flows in circular tubes of variable cross-sections, conical or similar geometry were made by Walicki et al. [26], Walicki and Walicka [27-29], Walicka and Walicki [30], Sochi [31-34], Walicka [35, 36].

It has been found that at the bottom of rivers, lakes, seas and oceans an enhanced transport of solutes and particulate matter can be encountered in a thin layer, which comprises a tiny portion of the seawater layer from top and a tiny portion of the porewater layer from below, called a benthic layer. In this layer there may exist an interaction between the fluid flow and living media as in bioreactors. The physicochemical and biological processes ongoing in the benthic layer cause that the fluid flowing through this layer behaves as a non-Newtonian fluid.

Flows of non-Newtonian fluids through porous media are frequently encountered in the petroleum industry (Vossoughi [37], Pearson and Tardy [38], Perrin et al. [39]). In exploitation of oil beds, an injection of polymer solutions into oil reservoirs is frequently applied to enhance oil recovery. Sometimes to achieve this aim, suspensions (frequently called slurries) of oil, coal and water are used (Vossoughi and Al-Husaini [40]).

The aim of this paper is to present an analytical method for deriving mathematical relations between the volumetric flow rate and pressure drop or pressure gradient in tapered-expanded or corrugated capillary fissures and tubes, such as those shown schematically in Fig.1. Employing the results of the recent papers by Walicka $[35,36]$, we present five examples of flows both in capillary fissures and tubes for non-Newtonian fluids of DeHaven type [41, 42]. Each of the examples under consideration finishes with formulae describing the velocities in a thin porous layer consisting of variable cross-section capillaries. The real layer is replaced with an equivalent matrix composed of homogeneous capillaries of constant cross-section for which the velocities are given. To take into account the real values of the velocities correction coefficients called "hindrance factors" were introduced. These flows may be used to model the classic flows through porous media [43] or to study PNMs [36].

\section{Fluids and capillaries description}

DeHaven fluids are pseudoplastic fluids which are characterized by a non-linear relationship between the shear stress and the shear strain rate; to be more precise it can be stated that the shear strain rate is a non-linear function of the shear stress [41, 42].

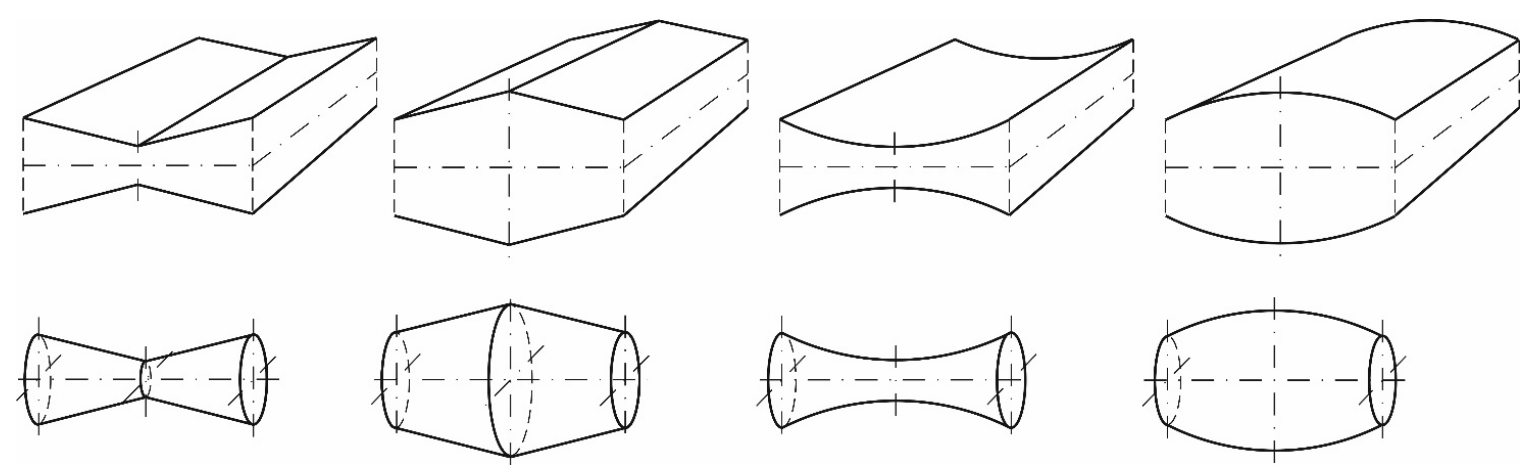

Fig.1. Capillary fissures or tubes: having convergent-divergent or divergent-convergent profiles. 
There are many models of fluids which, for suitably selected material coefficients, reduce to the model of the DeHaven fluid. Some of these models are presented in Table 1.1.

Table 1.1. Models of fluids similar to the DeHaven fluid model

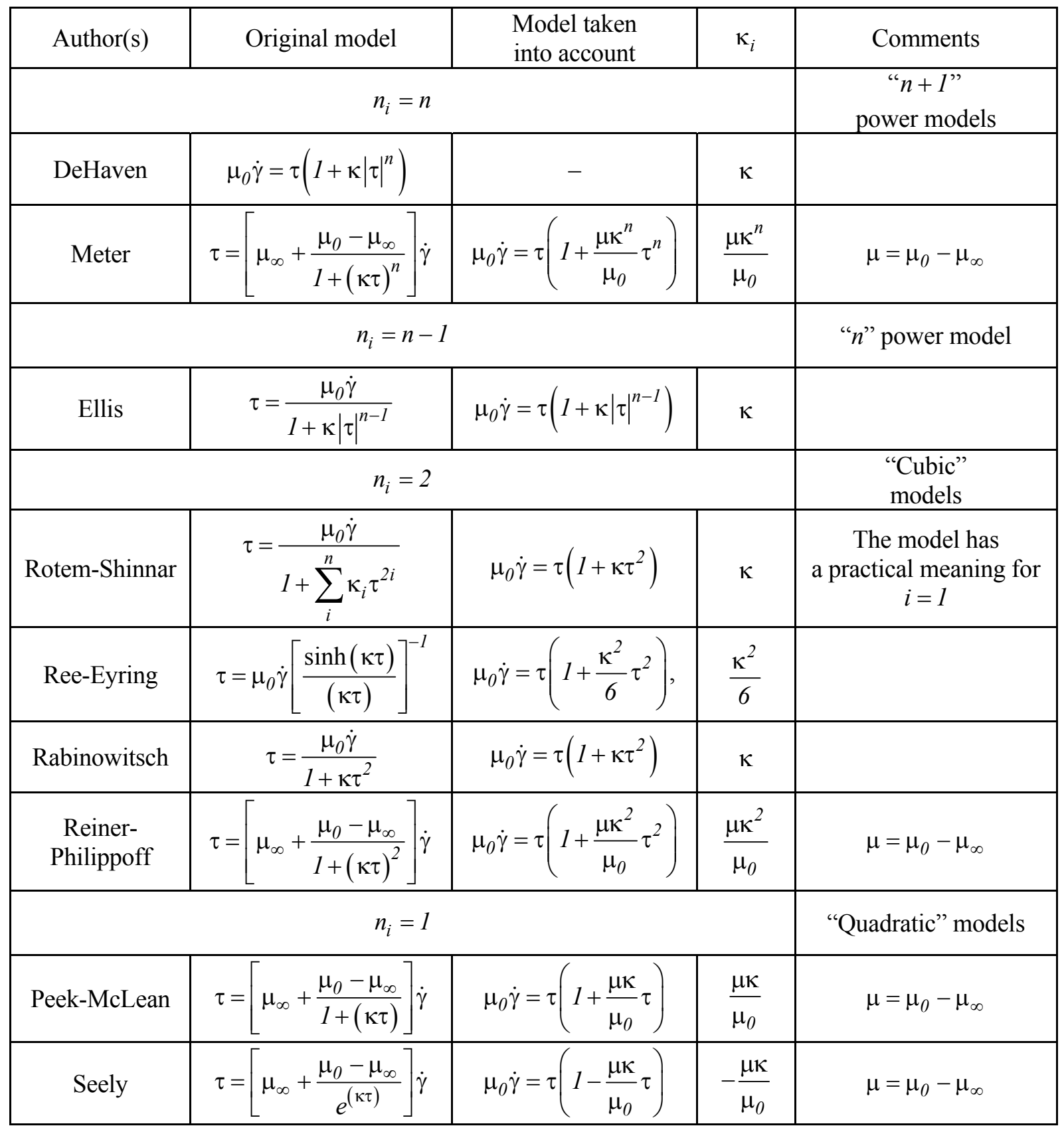

In what follows we will use the DeHaven fluid model to describe the flows through capillary fissures or tubes. The presence of capillary fissures in porous media seems to be extraordinary. Note that in exploitation of the oil beds one runs frequently into rocks rendered porous by solution or by fracturing. These interstices have a form of capillary fissures [43].

The capillaries being the subject of our considerations are presented schematically in Fig.1. Each of these capillaries will be described exactly in subsequent sections of this paper. 


\section{Flows through rectilinear capillary fissures or tubes with variable cross-section}

Frequently, rectilinear fissures or tubes of constant cross-sections are used to model the flow through porous media (Fig.2).

a)

b)
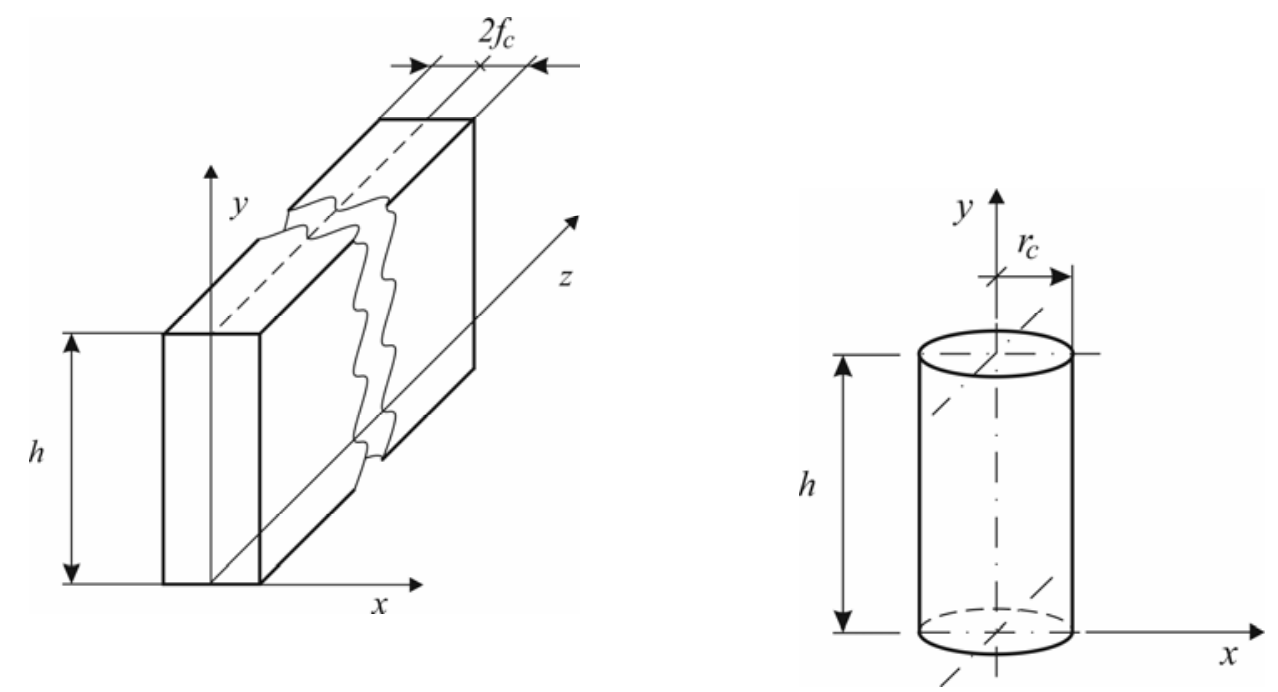

Fig.2. Geometry of a rectilinear capillary fissure (a) and a capillary tube (b) of constant cross-section.

The flow velocities of the DeHaven fluid in capillaries are given by the following expressions [43]: in the capillary fissure

$$
\mathrm{v}_{f}=\frac{f_{c}^{2}}{3 \mu}\left[1+\frac{3 k_{i} f_{c}^{n_{i}}}{\left(n_{i}+3\right)}\left(-\frac{d p}{d y}\right)^{n_{i}}\right]\left(-\frac{d p}{d y}\right)
$$

in the capillary tube

$$
\mathrm{v}_{t}=\frac{r_{c}^{2}}{8 \mu}\left[1+\frac{4 k_{i} r_{c}^{n_{i}}}{2^{n_{i}}\left(n_{i}+4\right)}\left(-\frac{d p}{d y}\right)^{n_{i}}\right]\left(-\frac{d p}{d y}\right) .
$$

The flow rates are, respectively, equal to:

- for the capillary fissure,

$$
Q_{f}=2 f_{c} \mathrm{v}_{f}=\frac{2 f_{c}^{3}}{3 \mu}\left[1+\frac{3 k_{i} f_{c}^{n_{i}}}{\left(n_{i}+3\right)}\left(-\frac{d p}{d y}\right)^{n_{i}}\right]\left(-\frac{d p}{d y}\right),
$$

here $Q_{f}$ is counted on the unit of a fissure width;

- for the capillary tube

$$
Q_{t}=\pi r_{c}^{2} \mathrm{v}_{t}=\frac{\pi r_{c}^{4}}{8 \mu}\left[1+\frac{4 k_{i} r_{c}^{n_{i}}}{2^{n_{i}}\left(n_{i}+4\right)}\left(-\frac{d p}{d y}\right)^{n_{i}}\right]\left(-\frac{d p}{d y}\right) .
$$


Assuming the principle of superposition we may write

$$
Q_{c}=Q_{N}+Q_{D H}
$$

where $Q_{c}$ is either $Q_{f}$ or $Q_{t}, Q_{N}$ is a Newtonian flow rate, $Q_{D H}$ denotes an additional flow rate connected with the DeHaven fluid.

Thus, we have, respectively

$$
Q_{f}=Q_{f N}+Q_{f D H}
$$

where

$$
\begin{aligned}
& Q_{f N}=\frac{2 f_{c}^{3}}{3 \mu}\left(-\frac{d p}{d y}\right) \\
& Q_{f D H}=\frac{2 k_{i} f_{c}^{n_{i}+3}}{\mu\left(n_{i}+3\right)}\left(-\frac{d p}{d y}\right)^{n_{i}+1}
\end{aligned}
$$

and

$$
\begin{aligned}
& Q_{t}=Q_{t N}+Q_{t D H} \\
& Q_{t N}=\frac{\pi r_{c}^{4}}{8 \mu}\left(-\frac{d p}{d y}\right), \\
& Q_{t D H}=\frac{\pi k_{i} r_{c}^{n_{i}+4}}{2^{n_{i}+1} \mu\left(n_{i}+4\right)}\left(-\frac{d p}{d y}\right)^{n_{i}+1} .
\end{aligned}
$$

To find the pressure drop in the flow through capillaries of variable cross-section (Fig.3) we have the following expressions:

- for the capillary fissure

$$
\begin{aligned}
& \Delta p_{f N}=\frac{3 \mu Q_{f N}}{2} \int_{-h / 2}^{h / 2} \frac{d y}{f_{c}^{3}}, \\
& \Delta p_{f D H}=\left[\frac{\mu\left(n_{i}+3\right) Q_{f D H}}{2 k_{i}}\right]^{\frac{1}{n_{i}+1}} \int_{-h / 2}^{h / 2} \frac{d y}{\frac{n_{i}+3}{f_{c}+1}},
\end{aligned}
$$

- for the capillary tube

$$
\begin{aligned}
& \Delta p_{t N}=\frac{8 \mu Q_{t N}}{\pi} \int_{-h / 2}^{h / 2} \frac{d y}{r_{c}^{4}}, \\
& \Delta p_{t D H}=2\left[\frac{\mu\left(n_{i}+4\right) Q_{t D H}}{\pi k_{i}}\right]^{\frac{1}{n_{i}+1}} \int_{-h / 2}^{h / 2} \frac{d y}{r_{c}^{\frac{n_{i}+4}{n_{i}+1}}},
\end{aligned}
$$



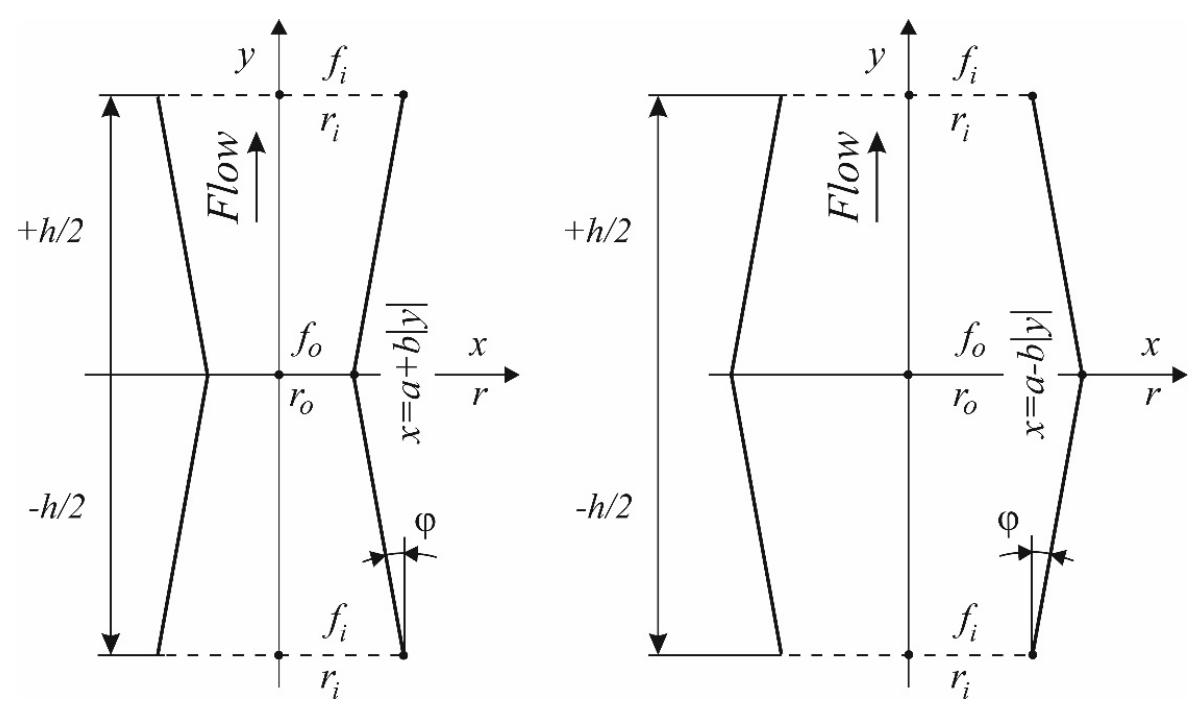

Fig.3. Scheme of convergent-divergent and divergent-convergent capillaries with rectilinear generatrices.

The current thickness of the capillary fissure or the radius of the capillary tube are given, respectively, by

$$
\left.\begin{array}{l}
f_{c}(y) \\
r_{c}(y)
\end{array}\right\}=x=a \pm b|y| \quad \text { where } \quad-\frac{h}{2} \leq y \leq+\frac{h}{2}
$$

and there are, respectively

$$
a=\left\{\begin{array}{l}
f_{o} \\
r_{o}
\end{array}, \quad b=\left\{\begin{array}{l} 
\pm \frac{2\left(f_{i}-f_{o}\right)}{h} \\
\pm \frac{2\left(r_{i}-r_{o}\right)}{h}
\end{array} .\right.\right.
$$

Introducing formulae (3.16) and (3.17) into Eqs (3.12),(3.13) or (3.14),(3.15) we will obtain - after integration - the following expressions (see Eqs (A.3)-(A.4) in the Appendix):

- for the capillary fissure

$$
\begin{aligned}
& \Delta p_{f N}=\frac{3 \mu Q_{f N}}{2} \frac{h\left[ \pm\left(f_{o}^{-2}-f_{i}^{-2}\right)\right]}{2\left[ \pm\left(f_{i}-f_{o}\right)\right]}, \\
& \Delta p_{f D H}=\left[\frac{\left(n_{i}+3\right) \mu Q_{f D H}}{2 k_{i}}\right]^{\frac{1}{n_{i}+1}} \frac{\left(n_{i}+1\right) h\left[ \pm\left(f_{o}^{-\frac{2}{n_{i}+1}}-f_{i}^{-\frac{2}{n_{i}+1}}\right)\right]}{2\left[ \pm\left(f_{i}-f_{o}\right)\right]}
\end{aligned}
$$

and

$$
Q_{f N}=\frac{4\left[ \pm\left(f_{i}-f_{o}\right)\right]}{3 \mu\left[ \pm\left(f_{o}^{-2}-f_{i}^{-2}\right)\right]}\left(\frac{\Delta p_{f N}}{h}\right)
$$




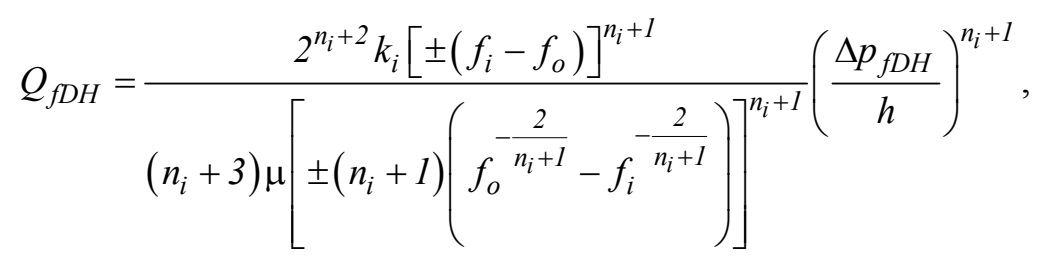

taking into account that

$$
\Delta p_{f N}=\Delta p_{f D H}=\Delta p
$$

and reusing the principle of superposition (3.6), we may write

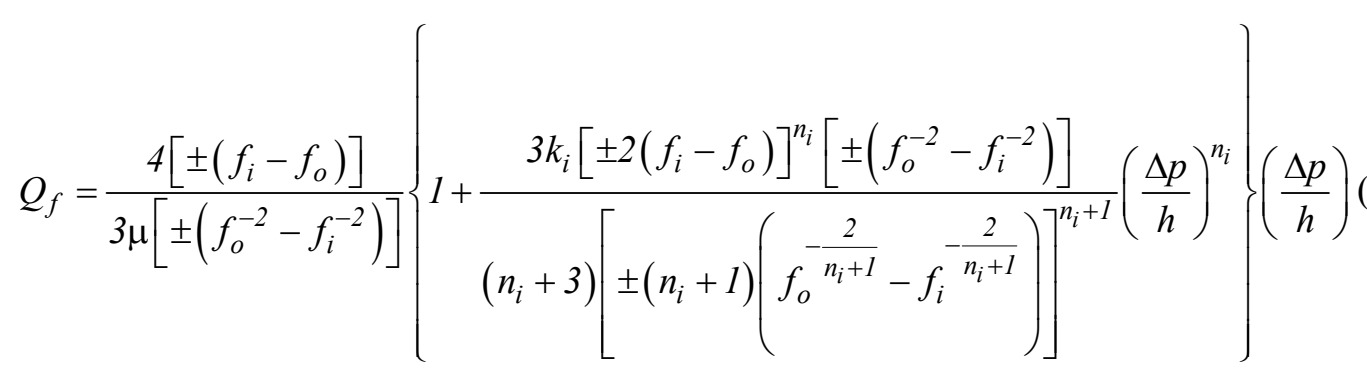

- for the capillary tube

$$
\begin{aligned}
& \Delta p_{t N}=\frac{8 \mu Q_{t N}}{3 \pi} \frac{h\left[ \pm\left(r_{o}^{-3}-r_{i}^{-3}\right)\right]}{\left[ \pm\left(r_{i}-r_{o}\right)\right]} \\
& \Delta p_{t D H}=\left[\frac{\left(n_{i}+4\right) \mu Q_{t D H}}{\pi k_{i}}\right]^{\frac{1}{n_{i}+1}} \frac{2\left(n_{i}+1\right) h\left[ \pm\left(r_{o}^{-\frac{3}{n_{i}+1}}-r_{i}^{-\frac{3}{n_{i}+1}}\right)\right]}{3\left[ \pm\left(r_{i}-r_{o}\right)\right]},
\end{aligned}
$$

and

$$
\begin{aligned}
& Q_{t N}=\frac{3 \pi\left[ \pm\left(r_{i}-r_{o}\right)\right]}{8 \mu\left[ \pm\left(r_{o}^{-3}-r_{i}^{-3}\right)\right]}\left(\frac{\Delta p_{t N}}{h}\right), \\
& Q_{t D H}=\frac{3^{n_{i}+1} k_{i} \pi\left[ \pm\left(r_{i}-r_{o}\right)\right]^{n_{i}+1}}{2^{n_{i}+1}\left(n_{i}+4\right) \mu\left[ \pm\left(n_{i}+1\right)\left(r_{o}^{-\frac{3}{n_{i}+1}}-r_{i}^{-\frac{3}{n_{i}+1}}\right)\right]^{n_{i}+1}\left(\frac{\Delta p_{t D H}}{h}\right)^{n_{i}+1},},
\end{aligned}
$$

taking into account that

$$
\Delta p_{t N}=\Delta p_{t D H}=\Delta p
$$

and reusing the principle of superposition (3.9), we may write: 


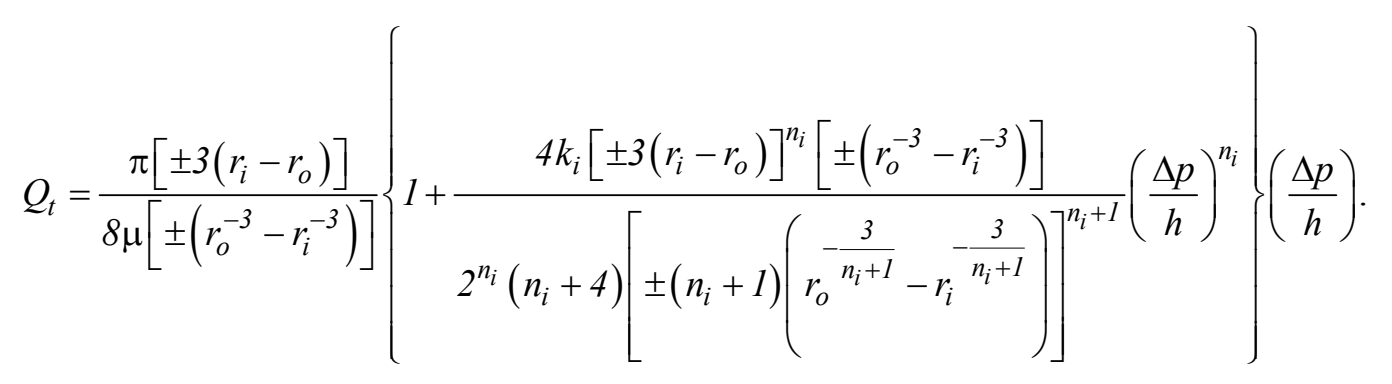

The flow velocities through a thin porous layer, composed of variable cross-section capillaries and modelled as an equivalent matrix of constant cross-section capillaries, will be given, respectively, as

$$
\begin{aligned}
& \mathrm{v}_{f}=\frac{Q_{f} \varphi_{p}}{2 f_{M}}=\frac{f_{M}^{2} \varphi_{p} \Psi_{1 f v}}{3 \mu}\left\{1+\frac{3 k_{i} f_{M}^{n_{i}} \Psi_{2 f v}}{\left(n_{i}+3\right)}\left(-\frac{d p}{d y}\right)^{n_{i}}\right\}\left(-\frac{d p}{d y}\right), \\
& \mathrm{v}_{t}=\frac{Q_{t} \varphi_{p}}{\pi r_{M}^{2}}=\frac{r_{M}^{2} \varphi_{p} \Psi_{1 t v}}{8 \mu}\left\{1+\frac{4 k_{i} r_{M}^{n_{i}} \psi_{2 t v}}{2^{n_{i}}\left(n_{i}+4\right)}\left(-\frac{d p}{d y}\right)^{n_{i}}\right\}\left(-\frac{d p}{d y}\right)
\end{aligned}
$$

where

$$
\begin{gathered}
\psi_{1 f v}=\frac{\left[ \pm 2\left(f_{i}-f_{o}\right)\right]}{f_{M}^{3}\left[ \pm\left(f_{o}^{-2}-f_{i}^{-2}\right)\right]}, \quad \psi_{2 f v}=\frac{\left[ \pm 2\left(f_{i}-f_{o}\right)\right]^{n_{i}}\left[ \pm\left(f_{o}^{-2}-f_{i}^{-2}\right)\right]}{f_{M}^{n_{i}}\left[ \pm\left(n_{i}+1\right)\left(f_{o}^{-\frac{2}{n_{i}+1}}-f_{i}^{-\frac{2}{n_{i}+1}}\right)\right]^{n_{i}+1}}, \\
\psi_{l t v}=\frac{\left[ \pm 3\left(r_{i}-r_{o}\right)\right]}{r_{M}^{4}\left[ \pm\left(r_{o}^{-3}-r_{i}^{-3}\right)\right]}, \quad \psi_{2 t v}=\frac{\left[ \pm 3\left(r_{i}-r_{o}\right)\right]^{n_{i}}\left[ \pm\left(r_{o}^{-3}-r_{i}^{-3}\right)\right]}{r_{M}^{n_{i}}\left[ \pm\left(n_{i}+1\right)\left(r_{o}^{-\frac{3}{n_{i}+1}}-r_{i}^{-\frac{3}{n_{i}+1}}\right)\right]^{n_{i}+1}},
\end{gathered}
$$

and $\varphi_{p}$ is the porosity of the porous layer, $\psi_{1 f v}, \psi_{l t v}$ are the first hindrance factors, $\psi_{2 f v}, \psi_{2 t v}$ are the second hindrance factors for the capillaries of variable cross-sections. Here, the index $M$ indicates maximum values $f_{i}, f_{o}$ or $r_{i}, r_{o}$ which correspond to the capillary thickness or capillary radius for the equivalent capillaries of constant cross-section.

\section{Flows through parabolic capillaries}

Parabolic capillaries, depicted in Fig.4, are described by the formulae

$$
\left.\begin{array}{l}
f_{c}(y) \\
r_{c}(y)
\end{array}\right\}=a \pm b y^{2} \quad \text { where } \quad-\frac{h}{2} \leq y \leq+\frac{h}{2}
$$

and 


$$
a=\left\{\begin{array}{l}
f_{o} \\
r_{o}
\end{array}, \quad b=\left\{\begin{array}{l} 
\pm\left(\frac{2}{h}\right)^{2}\left(f_{i}-f_{o}\right) \\
\pm\left(\frac{2}{h}\right)^{2}\left(r_{i}-r_{o}\right)
\end{array} .\right.\right.
$$
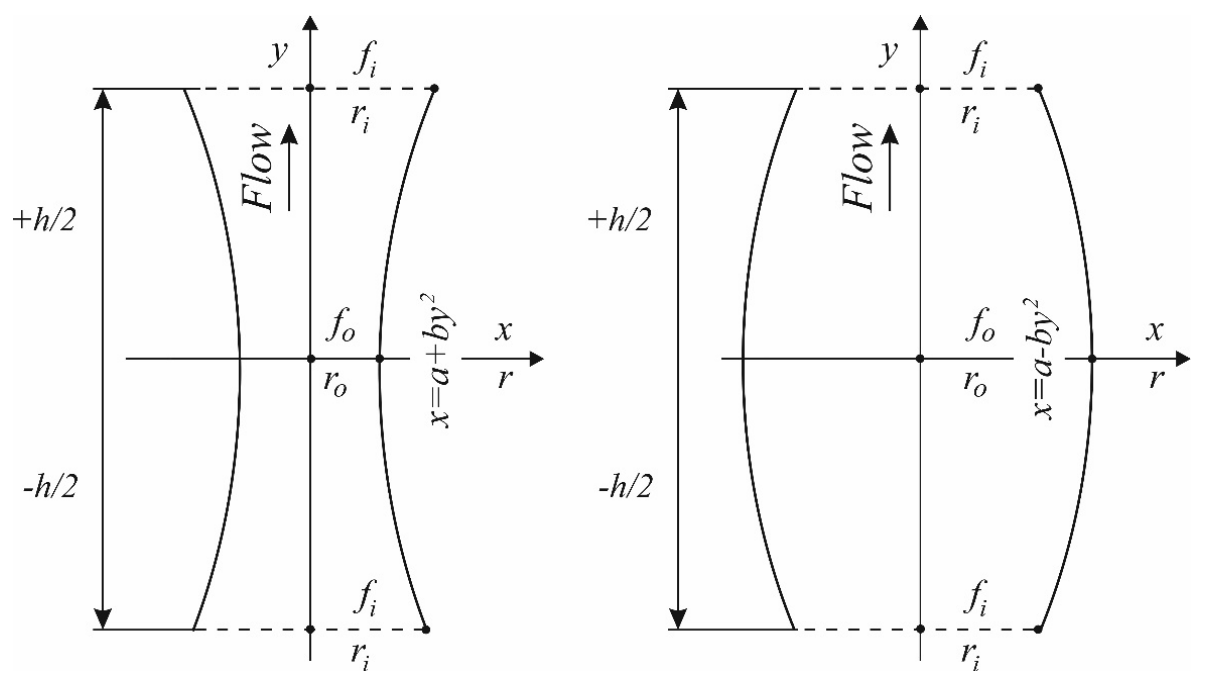

Fig.4. Schematic representation of convergent-divergent and divergent-convergent capillaries with parabolic profiles.

Introducing formulae (4.1) and (4.2) into Eqs (3.12),(3.13) or (3.14),(3.15) we will obtain - after integration - the following expressions (see Eqs (A.7), (A.9), (A.11) and (A.13) in the Appendix):

- for the capillary fissure

$$
\begin{aligned}
& \Delta p_{f N}=\frac{3 \mu Q_{f N} h}{2} F_{p N}, \\
& \Delta p_{f D H}=\left[\frac{\left(n_{i}+3\right) \mu Q_{f D H}}{2 k_{i}}\right]^{\frac{1}{n_{i}+1}} \frac{h}{f_{o}^{\frac{n_{i}+3}{n_{i}+1}}} F_{f p D H},
\end{aligned}
$$

and

$$
\begin{aligned}
& Q_{f N}=\frac{2}{3 \mu F_{p N}}\left(\frac{\Delta p_{f N}}{h}\right), \\
& Q_{f D H}=\frac{2 k_{i} f_{o}^{n_{i}+3}}{\left(n_{i}+3\right) \mu} \frac{1}{\left(F_{f p D H}\right)^{n_{i}+1}}\left(\frac{\Delta p_{f D H}}{h}\right)^{n_{i}+1}
\end{aligned}
$$

where

$$
F_{p N}=\frac{1}{4 f_{i}^{2} f_{o}}+\frac{3}{8 f_{i} f_{o}^{2}}+\frac{3}{8 f_{o}^{2}} J( \pm h),
$$




$$
\begin{aligned}
& J( \pm h)=\frac{2}{\left[ \pm f_{o}\left(f_{i}-f_{o}\right)\right]^{1 / 2}} \times\left\{\begin{array}{l}
\arctan \left(\frac{f_{i}-f_{o}}{f_{o}}\right)^{1 / 2} \\
\operatorname{arctanh}\left(\frac{f_{o}-f_{i}}{f_{o}}\right)^{1 / 2}
\end{array}\right. \\
& F_{f p D H}=F\left[\frac{1}{2}, \frac{n_{i}+3}{n_{i}+1} ; \frac{3}{2} ; \mp\left(1-\frac{f_{i}}{f_{o}}\right)\right] ;
\end{aligned}
$$

- for the capillary tube

$$
\begin{aligned}
& \Delta p_{t N}=\frac{8 \mu Q_{t N} h}{\pi} T_{p N}, \\
& \Delta p_{t D H}=\left[\frac{\left(n_{i}+4\right) \mu Q_{t D H}}{\pi k_{i}}\right]^{\frac{1}{n_{i}+1}} \frac{2 h}{\frac{n_{i}+4}{n_{i}+1}} F_{t p D H},
\end{aligned}
$$

and

$$
\begin{aligned}
& Q_{t N}=\frac{\pi}{8 \mu T_{p N}}\left(\frac{\Delta p_{t N}}{h}\right) \\
& Q_{t D H}=\frac{\pi k_{i} r_{o}^{n_{i}+4}}{2^{n_{i}+1}\left(n_{i}+4\right) \mu} \frac{1}{\left(F_{t p D H}\right)^{n_{i}+1}}\left(\frac{\Delta p_{t D H}}{h}\right)^{n_{i}+1}
\end{aligned}
$$

where

$$
\begin{aligned}
& T_{p N}=\frac{1}{6 r_{i}^{3} r_{o}}+\frac{5}{24 r_{i}^{2} r_{o}^{2}}+\frac{5}{16 r_{i} r_{o}^{3}}+\frac{5}{16 r_{o}^{3}} J( \pm h) \\
& J( \pm h)=\frac{2}{\left[ \pm r_{o}\left(r_{i}-r_{o}\right)\right]^{1 / 2}} \times\left\{\begin{array}{l}
\arctan \left(\frac{r_{i}-r_{o}}{r_{o}}\right)^{1 / 2} \\
\operatorname{arctanh}\left(\frac{r_{o}-r_{i}}{r_{o}}\right)^{1 / 2},
\end{array}\right. \\
& F_{t p D H}=F\left[\frac{1}{2}, \frac{n_{i}+4}{n_{i}+1} ; \frac{3}{2} ; \mp\left(1-\frac{r_{i}}{r_{o}}\right)\right] .
\end{aligned}
$$

The functions $F[\ldots]$ are so called "hypergeometric functions" and they are defined in the Appendix.

Taking into account Eqs (3.22) and (3.28) and reusing the principle of superposition (3.6) or (3.9), we may write 


$$
Q_{f}=\frac{2}{3 \mu F_{p N}}\left\{1+\frac{3 k_{i} f_{o}^{n_{i}+3} F_{p N}}{\left(n_{i}+3\right)\left(F_{f p D H}\right)^{n_{i}+1}}\left(\frac{\Delta p}{h}\right)^{n_{i}}\right\}\left(\frac{\Delta p}{h}\right),
$$

or

$$
Q_{t N}=\frac{\pi}{8 \mu T_{p N}}\left\{1+\frac{4 k_{i} r_{o}^{n_{i}+4} T_{p N}}{2^{n_{i}}\left(n_{i}+4\right)\left(F_{t p D H}\right)^{n_{i}+1}}\left(\frac{\Delta p}{h}\right)^{n_{i}}\right\}\left(\frac{\Delta p}{h}\right)
$$

The flow velocities through the thin porous layer composed of parabolic capillaries, modelled as the equivalent matrix of constant cross-section capillaries, will be given, respectively, as

$$
\begin{aligned}
& \mathrm{v}_{f}=\frac{Q_{f} \varphi_{p}}{2 f_{M}}=\frac{f_{M}^{2} \varphi_{p} \Psi_{1 f p}}{3 \mu}\left\{1+\frac{3 k_{i} f_{M}^{n_{i}} \Psi_{2 f p}}{\left(n_{i}+3\right)}\left(-\frac{d p}{d y}\right)^{n_{i}}\right\}\left(-\frac{d p}{d y}\right), \\
& \mathrm{v}_{t}=\frac{Q_{t} \varphi_{p}}{\pi r_{M}^{2}}=\frac{r_{M}^{2} \varphi_{p} \psi_{1 t p}}{8 \mu}\left\{1+\frac{4 k_{i} r_{M}^{n_{i}} \psi_{2 t p}}{2^{n_{i}}\left(n_{i}+4\right)}\left(-\frac{d p}{d y}\right)^{n_{i}}\right\}\left(-\frac{d p}{d y}\right)
\end{aligned}
$$

where

$$
\begin{aligned}
& \psi_{1 f p}=\frac{1}{f_{M}^{3} F_{p N}}, \quad \psi_{2 f p}=\left(\frac{f_{o}}{f_{M}}\right)^{n_{i}} \frac{f_{o}^{3} F_{p N}}{\left(F_{f p D H}\right)^{n_{i}+1}}, \\
& \psi_{1 t p}=\frac{1}{r_{M}^{4} T_{p N}}, \quad \psi_{2 t p}=\left(\frac{r_{o}}{r_{M}}\right)^{n_{i}} \frac{r_{o}^{4} T_{p N}}{\left(F_{t p D H}\right)^{n_{i}+1}}
\end{aligned}
$$

and $\psi_{1 f p}, \psi_{1 t p}$ are the first hindrance factors, $\psi_{2 f p}, \psi_{2 t p}$ are the second hindrance factors for the parabolic capillaries; the sense of the index $M$ is the same as in the previous section.

\section{Flows through hyperbolic capillaries}

For capillaries of hyperbolic profiles, similar to the profiles shown in Fig.4, the geometric description is as follows

$$
\left.\begin{array}{l}
f_{c}(y) \\
r_{c}(y)
\end{array}\right\}=\left(a \pm b y^{2}\right)^{1 / 2} \quad \text { where } \quad-\frac{h}{2} \leq y \leq+\frac{h}{2},
$$

and

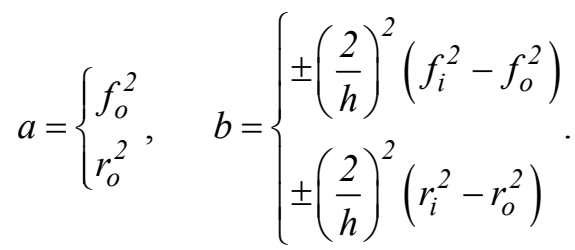

Introducing formulae (5.1) and (5.2) into Eqs (3.12), (3.13) or (3.14), (3.15) we will obtain - the expressions (see Eqs (A.15), (A.17) and (A.19) in the Appendix): 
- for the capillary fissure

$$
\begin{aligned}
& \Delta p_{f N}=\frac{3 \mu Q_{f N} h}{2 f_{i} f_{o}^{2}}, \\
& \Delta p_{f D H}=\left[\frac{\left(n_{i}+3\right) \mu Q_{f D H}}{2 k_{i}}\right]^{\frac{1}{n_{i}+1}} \frac{h}{\frac{n_{i}+3}{n_{i}+1}} F_{f h D H},
\end{aligned}
$$

and

$$
\begin{aligned}
& Q_{f N}=\frac{2 f_{i} f_{o}^{2}}{3 \mu}\left(\frac{\Delta p_{f N}}{h}\right), \\
& Q_{f D H}=\frac{2 k_{i} f_{o}^{n_{i}+3}}{\left(n_{i}+3\right) \mu} \frac{1}{\left(F_{f h D H}\right)^{n_{i}+1}}\left(\frac{\Delta p_{f D H}}{h}\right)^{n_{i}+1},
\end{aligned}
$$

where

$$
F_{f h D H}=F\left[\frac{1}{2}, \frac{n_{i}+3}{2\left(n_{i}+1\right)} ; \frac{3}{2} ; \pm\left(1-\frac{f_{i}^{2}}{f_{o}^{2}}\right)\right]
$$

- for the capillary tube

$$
\begin{aligned}
& \Delta p_{t N}=\frac{8 \mu Q_{t N} h}{\pi} T_{p}, \\
& \Delta p_{t D H}=\left[\frac{\left(n_{i}+4\right) \mu Q_{t D H}}{\pi k_{i}}\right]^{\frac{1}{n_{i}+1}} \frac{2 h}{\frac{n_{i}+4}{n_{i}+1}} F_{t h D H},
\end{aligned}
$$

and

$$
\begin{aligned}
& Q_{t N}=\frac{\pi}{8 \mu T_{p}}\left(\frac{\Delta p_{t N}}{h}\right) \\
& Q_{t D H}=\frac{\pi k_{i} r_{o}^{n_{i}+4}}{2^{n_{i}+1}\left(n_{i}+4\right) \mu} \frac{1}{\left(F_{t h D H}\right)^{n_{i}+1}}\left(\frac{\Delta p_{t D H}}{h}\right)^{n_{i}+1}
\end{aligned}
$$

where

$$
\begin{aligned}
& T_{p}=\frac{1}{2 r_{i}^{2} r_{o}^{2}}+\frac{1}{2 r_{o}^{2}} J( \pm h) \\
& F_{t h D H}=F\left[\frac{1}{2}, \frac{n_{i}+4}{2\left(n_{i}+1\right)} ; \frac{3}{2} ; \pm\left(1-\frac{r_{i}^{2}}{r_{o}^{2}}\right)\right] .
\end{aligned}
$$


where now

$$
J( \pm h)=\frac{2}{\left[ \pm r_{o}^{2}\left(r_{i}^{2}-r_{o}^{2}\right)\right]^{1 / 2}} \times\left\{\begin{array}{l}
\arctan \left(\frac{r_{i}^{2}-r_{o}^{2}}{r_{o}^{2}}\right)^{1 / 2} \\
\operatorname{arctanh}\left(\frac{r_{o}-r_{i}^{2}}{r_{o}^{2}}\right)^{1 / 2}
\end{array}\right.
$$

Taking into account Eqs (3.22) and (3.28) and reusing the principle of superposition (3.6) or (3.9), we may write the following expressions

$$
\begin{aligned}
& Q_{f}=\frac{2 f_{i} f_{o}^{2}}{3 \mu}\left\{1+\frac{3 k_{i} f_{o}^{n_{i}+1}}{f_{i}\left(n_{i}+3\right)\left(F_{f h D H}\right)^{n_{i}+1}}\left(\frac{\Delta p}{h}\right)^{n_{i}}\right\}\left(\frac{\Delta p}{h}\right), \\
& Q_{t}=\frac{\pi}{8 \mu T_{p}}\left\{1+\frac{4 k_{i} r_{o}^{n_{i}+4} T_{p}}{2^{n_{i}}\left(n_{i}+4\right)\left(F_{t h D H}\right)^{n_{i}+1}}\left(\frac{\Delta p}{h}\right)^{n_{i}}\right\}\left(\frac{\Delta p}{h}\right)
\end{aligned}
$$

The flow velocities through the thin porous layer composed of hyperbolic capillaries and modelled as the equivalent matrix of constant cross-section capillaries, will be given, respectively, as

$$
\begin{aligned}
& \mathrm{v}_{f}=\frac{Q_{f} \varphi_{p}}{2 f_{M}}=\frac{f_{M}^{2} \varphi_{p} \psi_{1 f h}}{3 \mu}\left\{1+\frac{3 k_{i} f_{M}^{n_{i}} \psi_{2 f h}}{\left(n_{i}+3\right)}\left(-\frac{d p}{d y}\right)^{n_{i}}\right\}\left(-\frac{d p}{d y}\right), \\
& \mathrm{v}_{t}=\frac{r_{M}^{2} \varphi_{p} \psi_{1 t h}}{8 \mu}\left\{1+\frac{4 k_{i} r_{M}^{n_{i}} \psi_{2 t h}}{2^{n_{i}}\left(n_{i}+4\right)}\left(-\frac{d p}{d y}\right)^{n_{i}}\right\}\left(-\frac{d p}{d y}\right)
\end{aligned}
$$

where

$$
\begin{aligned}
& \psi_{1 f h}=\left\{\begin{array}{l}
\left(\frac{f_{o}}{f_{M}}\right)^{2}, \\
\left(\frac{f_{i}}{f_{M}}\right)^{2}
\end{array} \psi_{2 f h}=\left\{\begin{array}{l}
\left(\frac{f_{o}}{f_{M}}\right)^{n_{i}+1} \frac{1}{\left(F_{f h D H}\right)^{n_{i}+1}} \\
\left(\frac{f_{i}}{f_{M}}\right)^{-1} \frac{1}{\left(F_{f h D H}\right)^{n_{i}+1}}
\end{array},\right.\right. \\
& \psi_{\text {lth }}=\frac{1}{r_{M}^{4} T_{p}}, \quad \Psi_{2 t h}=\left\{\begin{array}{l}
\left(\frac{r_{o}}{r_{M}}\right)^{n_{i}} \frac{r_{o}^{4} T_{p}}{\left(F_{t h D H}\right)^{n_{i}+1}} \\
\frac{r_{o}^{4} T_{p}}{\left(F_{t h D H}\right)^{n_{i}+1}}
\end{array},\right.
\end{aligned}
$$

and $\psi_{1 f h}, \psi_{1 t h}$ are the first hindrance factors, $\psi_{2 f h}, \psi_{2 t h}$ are the second hindrance factors for the hyperbolic capillaries. 


\section{Flows through hyperbolic cosine capillaries}

For capillaries of hyperbolic cosine profiles, similar to the profiles shown in Fig.4, the geometric description is given as follows

$$
\left.\begin{array}{l}
f_{c}(y) \\
r_{c}(y)
\end{array}\right\}=[a \cosh (b y)]^{ \pm 1} \quad \text { where } \quad-\frac{h}{2} \leq y \leq+\frac{h}{2}
$$

and

$$
a=\left\{\begin{array}{l}
f_{o}^{ \pm 1} \\
r_{o}^{ \pm 1},
\end{array}, \quad b=\left\{\begin{array}{l}
\left(\frac{2}{h}\right) \operatorname{arccosh}\left(\frac{f_{i}}{f_{o}}\right)^{ \pm l} \\
\left(\frac{2}{h}\right) \operatorname{arccosh}\left(\frac{r_{i}}{r_{o}}\right)^{ \pm 1}
\end{array} .\right.\right.
$$

Note that the exponent value equal to +1 is adequate to the convergent-divergent capillary in Fig.4, whereas the exponent value -1 is adequate to divergent-convergent capillary. Hence, Eqs (3.12), (3.13) or (3.14), (3.15) for the exponent value equal to +1 (see Eqs (A.20)-(A.25) in the Appendix) will become:

- for the capillary fissure

$$
\begin{aligned}
& \Delta p_{f N}=\frac{3 \mu Q_{f N} h}{4 f_{o}^{3} \operatorname{arccosh}\left(\frac{f_{i}}{f_{o}}\right)} F_{h N}, \\
& \Delta p_{f D H}=\left[\frac{\left(n_{i}+3\right) \mu Q_{f D H}}{2 k_{i}}\right]^{\frac{1}{n_{i}+1}} \frac{\left(n_{i}+1\right) h}{2 f_{o} f_{i}^{2 /\left(n_{i}+1\right)} \operatorname{arccosh}\left(\frac{f_{i}}{f_{o}}\right)} \operatorname{Im}\left(F_{f h D H}\right),
\end{aligned}
$$

and

$$
\begin{aligned}
& Q_{f N}=\frac{4 f_{o}^{3}\left[\operatorname{arccosh}\left(\frac{f_{i}}{f_{o}}\right)\right]}{3 \mu} \frac{1}{F_{h N}}\left(\frac{\Delta p_{f N}}{h}\right), \\
& Q_{f D H}=\frac{2^{n_{i}+2} k_{i} f_{o}^{n_{i}+1} f_{i}^{2}\left[\operatorname{arccosh}\left(\frac{f_{i}}{f_{o}}\right)\right]^{n_{i}+1}}{\left(n_{i}+1\right)^{n_{i}+1}\left(n_{i}+3\right) \mu} \frac{1}{\left[\operatorname{Im}\left(F_{f h D H}\right)\right]^{n_{i}+1}}\left(\frac{\Delta p_{f D H}}{h}\right)^{n_{i}+1}
\end{aligned}
$$

where

$$
\begin{aligned}
& F_{h N}=\frac{f_{o}^{2} \sinh \left[\operatorname{arccosh}\left(\frac{f_{i}}{f_{o}}\right)\right]}{f_{i}^{2}}+\arctan \left\{\sinh \left[\operatorname{arccosh}\left(\frac{f_{i}}{f_{o}}\right)\right]\right\} \\
& \operatorname{Im}\left(F_{f h D H}\right)=\operatorname{Im}\left\{F\left[\frac{1}{2},-\frac{1}{n_{i}+1} ; \frac{n_{i}}{n_{i}+1} ; \frac{f_{i}^{2}}{f_{o}^{2}}\right]\right\}
\end{aligned}
$$


- for the capillary tube

$$
\begin{aligned}
& \Delta p_{t N}=\frac{8 \mu Q_{t N} h}{\pi r_{o}^{4}\left[\operatorname{arccosh}\left(\frac{r_{i}}{r_{o}}\right)\right]} T_{h N}, \\
& \Delta p_{t D H}=\left[\frac{\left(n_{i}+4\right) \mu Q_{t D H}}{\pi k_{i}}\right]^{\frac{1}{n_{i}+1}} \frac{2\left(n_{i}+1\right) h}{3 r_{o} r_{i}^{3 /\left(n_{i}+1\right)} \operatorname{arccosh}\left(\frac{r_{i}}{r_{o}}\right)} \operatorname{Im}\left(F_{t h D H}\right),
\end{aligned}
$$

and

$$
\begin{aligned}
Q_{t N} & =\frac{\pi r_{o}^{4}\left[\operatorname{arccosh}\left(\frac{r_{i}}{r_{o}}\right)\right]}{8 \mu} \frac{1}{T_{h N}}\left(\frac{\Delta p_{t N}}{h}\right), \\
Q_{t D H} & =\frac{3^{n_{i}+1} \pi k_{i} r_{o}^{n_{i}+1} r_{i}^{3}\left[\operatorname{arccosh}\left(\frac{r_{i}}{r_{o}}\right)\right]^{n_{i}+1}}{2^{n_{i}+1}\left(n_{i}+1\right)^{n_{i}+1}\left(n_{i}+4\right) \mu} \frac{1}{\left[\operatorname{Im}\left(F_{t h D H}\right)\right]^{n_{i}+1}}\left(\frac{\Delta p_{f D H}}{h}\right)^{n_{i}+1}
\end{aligned}
$$

where

$$
\begin{aligned}
& T_{h N}=\left\{1-\frac{1}{3}\left(\tanh \left[\operatorname{arccosh}\left(\frac{r_{i}}{r_{o}}\right)\right]\right)^{2}\right\} \tanh \left[\operatorname{arccosh}\left(\frac{r_{i}}{r_{o}}\right)\right] . \\
& \operatorname{Im}\left(F_{t h D H}\right)=\operatorname{Im}\left\{F\left[\frac{1}{2},-\frac{3}{2\left(n_{i}+1\right)} ; \frac{2 n_{i}-1}{2\left(n_{i}+1\right)} ; \frac{r_{i}^{2}}{r_{o}^{2}}\right]\right\} .
\end{aligned}
$$

Here, $\operatorname{Im}(F)$ is the imaginary part of the hypergeometric function.

Taking into account Eqs (3.22) and (3.28) and reusing the principle of superposition (3.6) or (3.9), we can obtain

$$
\begin{aligned}
& Q_{f}=\frac{4 f_{o}^{3}\left[\operatorname{arccosh}\left(\frac{f_{i}}{f_{o}}\right)\right]}{3 \mu F_{h N}}\left\{1+\frac{3 \cdot 2^{n_{i}} k_{i} f_{o}^{n_{i}-2} f_{i}^{2}\left[\operatorname{arccosh}\left(\frac{f_{i}}{f_{o}}\right)\right]^{n_{i}} F_{h N}}{\left(n_{i}+1\right)^{n_{i}+1}\left(n_{i}+3\right)\left[\operatorname{Im}\left(F_{f h D H}\right)\right]^{n_{i}+1}}\left(\frac{\Delta p}{h}\right)^{n_{i}}\right\}\left(\frac{\Delta p}{h}\right), \\
& Q_{t}=\frac{\pi r_{o}^{4}\left[\operatorname{arccosh}\left(\frac{r_{i}}{r_{o}}\right)\right]}{8 \mu T_{h N}}\left\{1+\frac{4 \cdot 3^{n_{i}+1} k_{i} r_{o}^{n_{i}-3} r_{i}^{3}\left[\operatorname{arccosh}\left(\frac{r_{i}}{r_{o}}\right)\right]^{n_{i}} T_{h N}}{2^{n_{i}}\left(n_{i}+1\right)^{n_{i}+1}\left(n_{i}+4\right)\left[\operatorname{Im}\left(F_{t h D H}\right)\right]^{n_{i}+1}}\left(\frac{\Delta p}{h}\right)^{n_{i}}\right\}\left(\frac{\Delta p}{h}\right) .
\end{aligned}
$$


The flow velocities through the thin porous layer (for the exponent value equal to +1 ) modelled as the matrix of constant cross-section capillaries, will be given, respectively, as

$$
\begin{aligned}
& \mathrm{v}_{f}=\frac{f_{i}^{2} \varphi_{p} \Psi_{1 f h_{c}}}{3 \mu}\left\{1+\frac{3 k_{i} f_{i}^{n_{i}} \Psi_{2 f h_{c}}}{\left(n_{i}+3\right)}\left(-\frac{d p}{d y}\right)^{n_{i}}\right\}\left(-\frac{d p}{d y}\right), \\
& \mathrm{v}_{t}=\frac{r_{i}^{2} \varphi_{p} \psi_{1 t h_{c}}}{8 \mu}\left\{1+\frac{4 k_{i} r_{i}^{n_{i}} \psi_{2 t h_{c}}}{2^{n_{i}}\left(n_{i}+4\right)}\left(-\frac{d p}{d y}\right)^{n_{i}}\right\}\left(-\frac{d p}{d y}\right)
\end{aligned}
$$

where

$$
\begin{gathered}
\psi_{1 f h_{c}}=\frac{2 f_{o}^{3}\left[\operatorname{arccosh}\left(\frac{f_{i}}{f_{o}}\right)\right]}{f_{i}^{3} F_{h N}}, \quad \psi_{2 f h_{c}}=\frac{2^{n_{i}} f_{o}^{n_{i}-2}\left[\operatorname{arccosh}\left(\frac{f_{i}}{f_{o}}\right)\right]^{n_{i}} F_{h N}}{\left(n_{i}+1\right)^{n_{i}+1} f_{i}^{n_{i}-2}\left[\operatorname{Im}\left(F_{f h D H}\right)\right]^{n_{i}+1}}, \\
\psi_{1 t h_{c}}=\frac{r_{o}^{4}\left[\operatorname{arccosh}\left(\frac{r_{i}}{r_{o}}\right)\right]}{r_{i}^{4} T_{h N}}, \quad \psi_{2 t h_{c}}=\frac{3^{n_{i}+1} r_{o}^{n_{i}-3}\left[\operatorname{arccosh}\left(\frac{r_{i}}{r_{o}}\right)\right]^{n_{i}} T_{h N}}{\left(n_{i}+1\right)^{n_{i}+1} r_{i}^{n_{i}-3}\left[\operatorname{Im}\left(F_{t h D H}\right)\right]^{n_{i}+1}}
\end{gathered}
$$

and $\psi_{1 f h_{c}}, \psi_{1 t h_{c}}$ are the first hindrance factors, $\psi_{2 f h_{c}}, \psi_{2 t h_{c}}$ are the second hindrance factors for the hyperbolic cosine capillaries.

Equations (3.12), (3.13) or (3.14), (3.15), for the exponent value equal to -1 (see formulae (A.26)(A.31) in the Appendix) will become:

- for the capillary fissure

$$
\begin{aligned}
& \Delta p_{f N}=\frac{9 \mu Q_{f} h}{8 f_{o}^{3} \operatorname{arccosh}\left(\frac{f_{o}}{f_{i}}\right)} F_{h N}, \\
& \Delta p_{f D H}=\left[\frac{\left(n_{i}+3\right) \mu Q_{f D H}}{2 k_{i}}\right]^{\frac{1}{n_{i}+1}} \frac{\left(n_{i}+1\right) h f_{o}}{2\left(n_{i}+2\right) f_{i}^{\frac{2\left(n_{i}+2\right)}{n_{i}+1}} \operatorname{arccosh}\left(\frac{f_{o}}{f_{i}}\right)} \operatorname{Im}\left(F_{f h D H}\right),
\end{aligned}
$$

and

$$
\begin{aligned}
Q_{f N} & =\frac{8 f_{o}^{3}\left[\operatorname{arccosh}\left(\frac{f_{o}}{f_{i}}\right)\right]}{9 \mu} \frac{1}{F_{h N}}\left(\frac{\Delta p_{f N}}{h}\right), \\
Q_{f D H} & =\frac{2^{n_{i}+2}\left(n_{i}+2\right)^{n_{i}+1} k_{i} f_{i}^{2 n_{i}+4}\left[\operatorname{arccosh}\left(\frac{f_{o}}{f_{i}}\right)\right]^{n_{i}+1}}{\left(n_{i}+1\right)^{n_{i}+1}\left(n_{i}+3\right) \mu f_{o}^{n_{i}+1}\left[\operatorname{Im}\left(F_{f h D H}\right)\right]^{n_{i}+1}}\left(\frac{\Delta p_{f D H}}{h}\right)^{n_{i}+1}
\end{aligned}
$$


where now

$$
\begin{aligned}
& F_{h N}=\sinh \left[\operatorname{arccosh}\left(\frac{f_{o}}{f_{i}}\right)\right]+\frac{1}{9} \sinh \left[3 \operatorname{arccosh}\left(\frac{f_{o}}{f_{i}}\right)\right] \\
& \operatorname{Im}\left(F_{f h D H}\right)=\operatorname{Im}\left\{F\left[\frac{1}{2}, \frac{n_{i}+2}{n_{i}+1} ; \frac{2 n_{i}+3}{n_{i}+1} ;\left(\frac{f_{o}}{f_{i}}\right)^{2}\right]\right\} ;
\end{aligned}
$$

- for the capillary tube

$$
\begin{aligned}
\Delta p_{t N} & =\frac{\mu Q_{t} h}{\pi r_{o}^{4} \operatorname{arccosh}\left(\frac{r_{o}}{r_{i}}\right)} F_{t N}, \\
\Delta p_{t D H} & =\left[\frac{\left(n_{i}+4\right) \mu Q_{t D H}}{\pi k_{i}}\right]^{\frac{1}{n_{i}+1}} \frac{2\left(n_{i}+1\right) h r_{o}}{\left(2 n_{i}+5\right) r_{i}^{\frac{2 n_{i}+5}{n_{i}+1}} \operatorname{arccosh}\left(\frac{r_{i}}{r_{o}}\right)} \operatorname{Im}\left(F_{t h D H}\right),
\end{aligned}
$$

and

$$
\begin{aligned}
& Q_{t N}=\frac{\pi r_{o}^{4}\left[\arccos \left(\frac{r_{o}}{r_{i}}\right)\right]}{\mu} \frac{1}{F_{t N}}\left(\frac{\Delta p_{t N}}{h}\right), \\
& Q_{t D H}=\frac{\left(2 n_{i}+5\right)^{n_{i}+1} \pi k_{i} r_{i}^{2 n_{i}+5}\left[\operatorname{arccosh}\left(\frac{r_{o}}{r_{i}}\right)\right]^{n_{i}+1}}{\left(n_{i}+1\right)^{n_{i}+1}\left(n_{i}+4\right) \mu r_{o}^{n_{i}+1}\left[\operatorname{Im}\left(F_{t h D H}\right)\right]^{n_{i}+1}}\left(\frac{\Delta p_{t D H}}{h}\right)^{n_{i}+1}
\end{aligned}
$$

where now

$$
\begin{aligned}
& F_{t N}=3 \operatorname{arccosh}\left(\frac{r_{o}}{r_{i}}\right)+2 \sinh \left[2 \operatorname{arccosh}\left(\frac{r_{o}}{r_{i}}\right)\right]+\frac{1}{4} \sinh \left[4 \operatorname{arccosh}\left(\frac{r_{o}}{r_{i}}\right)\right] . \\
& \operatorname{Im}\left(F_{t h D H}\right)=\operatorname{Im}\left\{F\left[\frac{1}{2}, \frac{2 n_{i}+5}{2 n_{i}+2} ; \frac{4 n_{i}+7}{2 n_{i}+2} ;\left(\frac{r_{o}}{r_{i}}\right)^{2}\right]\right\} .
\end{aligned}
$$

Taking into account Eqs (3.22) and (3.28) and reusing the principle of superposition (3.6) or (3.9), we can write

$$
Q_{f}=\frac{8 f_{o}^{3}\left[\operatorname{arccosh}\left(\frac{f_{o}}{f_{i}}\right)\right]}{9 \mu F_{h N}}\left\{1+\frac{9 \cdot 2^{n_{i}-1}\left(n_{i}+2\right)^{n_{i}+1} k_{i} f_{i}^{2\left(n_{i}+2\right)}\left[\operatorname{arccosh}\left(\frac{f_{o}}{f_{i}}\right)\right]^{n_{i}} F_{h N}}{\left(n_{i}+1\right)^{n_{i}+1}\left(n_{i}+3\right) f_{o}^{n_{i}+4}\left[\operatorname{Im}\left(F_{f h D H}\right)\right]^{n_{i}+1}}\left(\frac{\Delta p}{h}\right)^{n_{i}}\right\}\left(\frac{\Delta p}{h}\right),
$$




$$
Q_{t}=\frac{\pi r_{o}^{4}\left[\arccos \left(\frac{r_{o}}{r_{i}}\right)\right]}{\mu F_{t N}}\left\{1+\frac{\left(2 n_{i}+5\right)^{n_{i}+1} k_{i} r_{i}^{2 n_{i}+5}\left[\operatorname{arccosh}\left(\frac{r_{o}}{r_{i}}\right)\right]^{n_{i}} F_{t N}}{\left(n_{i}+1\right)^{n_{i}+1}\left(n_{i}+4\right) r_{o}^{n_{i}+5}\left[\operatorname{Im}\left(F_{t h D H}\right)\right]^{n_{i}+1}}\left(\frac{\Delta p}{h}\right)^{n_{i}}\right\}\left(\frac{\Delta p}{h}\right)
$$

The flow velocities through the thin porous layer (for the exponent value equal to -1 ) modelled as the matrix of constant cross-section capillaries, will be given, respectively, as

$$
\begin{aligned}
& \mathrm{v}_{f}=\frac{f_{o}^{2} \varphi_{p} \Psi_{1 f h_{c}}}{3 \mu}\left\{1+\frac{3 k_{i} f_{o}^{n_{i}} \Psi_{2 f h_{c}}}{\left(n_{i}+3\right)}\left(-\frac{d p}{d y}\right)^{n_{i}}\right\}\left(-\frac{d p}{d y}\right), \\
& \mathrm{v}_{t}=\frac{r_{i}^{2} \varphi_{p} \Psi_{1 t h_{c}}}{8 \mu}\left\{1+\frac{4 k_{i} r_{i}^{n_{i}} \Psi_{2 t h_{c}}}{\left(n_{i}+4\right)}\left(-\frac{d p}{d y}\right)^{n_{i}}\right\}\left(-\frac{d p}{d y}\right)
\end{aligned}
$$

where

$$
\begin{gathered}
\psi_{1 f h_{c}}=\frac{4\left[\operatorname{arccosh}\left(\frac{f_{o}}{f_{i}}\right)\right]}{3 F_{h N}}, \quad \psi_{2 f h_{c}}=\frac{3 \cdot 2^{n_{i}-1}\left(n_{i}+2\right)^{n_{i}+1} f_{i}^{2 n_{i}+4}\left[\operatorname{arccosh}\left(\frac{f_{o}}{f_{i}}\right)\right]^{n_{i}} F_{h N}}{\left(n_{i}+1\right)^{n_{i}+1} f_{o}^{2 n_{i}+4}\left[\operatorname{Im}\left(F_{f h D H}\right)\right]^{n_{i}+1}}, \\
\psi_{1 t h_{c}}=\frac{8 r_{o}^{4}\left[\arccos \left(\frac{r_{o}}{r_{i}}\right)\right]}{r_{i}^{4} F_{t N}}, \quad \psi_{2 t h_{c}}=\frac{\left(2 n_{i}+5\right)^{n_{i}+1} r_{i}^{n_{i}+5}\left[\operatorname{arccosh}\left(\frac{r_{o}}{r_{i}}\right)\right]^{n_{i}} F_{t N}}{4\left(n_{i}+1\right)^{n_{i}+1} r_{o}^{n_{i}+5}\left[\operatorname{Im}\left(F_{t h D H}\right)\right]^{n_{i}+1}}
\end{gathered}
$$

and $\psi_{1 f h_{c}}, \psi_{1 t h_{c}}$ are the first hindrance factors, $\psi_{2 f h_{c}}, \psi_{2 t h_{c}}$ are the second hindrance factors for the hyperbolic cosine capillaries.

\section{Flows through cosine curve capillaries}

For capillaries of cosine curve profile, shown in Fig.5, where the capillary length $h$ spans one complete wavelength, the current capillary thickness or radius are given by

$$
\left.\begin{array}{l}
f_{c}(y) \\
r_{c}(y)
\end{array}\right\}=a \mp b \cos (k y) \quad \text { where } \quad-\frac{h}{2} \leq y \leq+\frac{h}{2}
$$

where

$$
a=\left\{\begin{array}{l}
\frac{f_{i}+f_{o}}{2} \\
\frac{r_{i}+r_{o}}{2}
\end{array}, \quad b=\left\{\begin{array}{l} 
\pm \frac{f_{i}-f_{o}}{2} \\
\pm \frac{r_{i}-r_{o}}{2}
\end{array} \quad \text { and } \quad k=\frac{2 \pi}{h}\right.\right.
$$



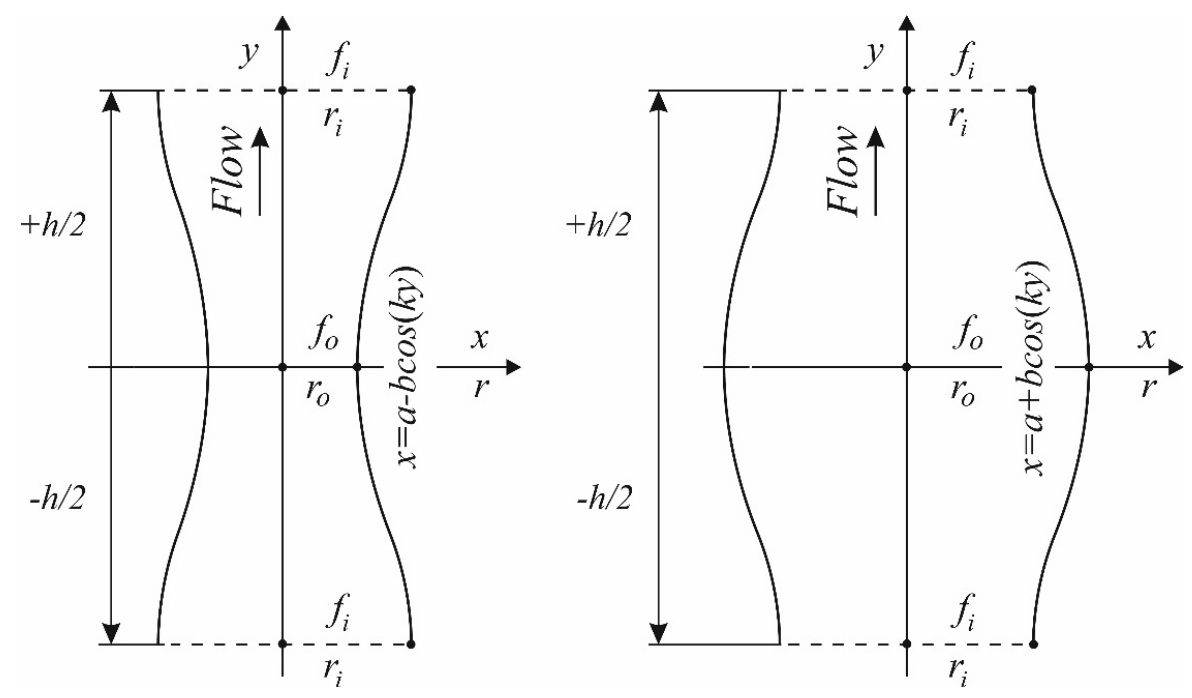

Fig.5. Schematic representation of convergent-divergent and divergent-convergent capillaries with a cosine curve profile.

Introducing formulae (7.1) and (7.2) into Eqs (3.12), (3.13) or (3.14), (3.15)-(3.6) we will obtain (see Eqs (A.32) $\div$ (A.40) in the Appendix):

- for the capillary fissure

$$
\begin{aligned}
& \Delta p_{f N}=\frac{3 \mu Q_{f N} h}{4} F_{c N}, \\
& \Delta p_{f D H}=\left[\frac{\left(n_{i}+3\right) \mu Q_{f D H}}{2 k_{i}}\right]^{\frac{1}{n_{i}+1}} \frac{\left(n_{i}+1\right) h}{2 \pi f_{M}^{2 /\left(n_{i}+1\right)}\left(f_{i} f_{o}\right)^{1 / 2}} \operatorname{Im}\left(F_{1 f h D H}\right),
\end{aligned}
$$

and

$$
\begin{aligned}
& Q_{f N}=\frac{4}{3 \mu} \frac{1}{F_{c N}}\left(\frac{\Delta p_{f N}}{h}\right), \\
& Q_{f D H}=\frac{2^{n_{i}+2} \pi^{n_{i}+1} k_{i} f_{M}^{2}\left(f_{i} f_{o}\right)^{\left(n_{i}+1\right) / 2}}{\left(n_{i}+1\right)^{n_{i}+1}\left(n_{i}+3\right) \mu} \frac{1}{\left[\operatorname{Im}\left(F_{l f h D H}\right)\right]^{n_{i}+1}}\left(\frac{\Delta p_{f D H}}{h}\right)^{n_{i}+1}
\end{aligned}
$$

where

$$
\begin{aligned}
& F_{c N}=\frac{3 f_{i}^{2}+2 f_{i} f_{o}+3 f_{o}^{2}}{\left(f_{i} f_{o}\right)^{5 / 2}} ; \\
& \operatorname{Im}\left(F_{1 f h D H}\right)=\operatorname{Im}\left\{F_{l}\left[-\frac{2}{n_{i}+1}, \frac{1}{2} ; \frac{1}{2} ; \frac{n_{i}-1}{n_{i}+1} ; 1,\left(\frac{f_{i}}{f_{o}}\right)^{ \pm 1}\right]\right\}
\end{aligned}
$$

- for the capillary tube 


$$
\begin{aligned}
& \Delta p_{t N}=\frac{2 \mu Q_{t N} h}{\pi} T_{c N}, \\
& \Delta p_{t D H}=\left[\frac{\left(n_{i}+4\right) \mu Q_{t D H}}{\pi k_{i}}\right]^{\frac{1}{n_{i}+1}} \frac{\left(n_{i}+1\right) h}{3 \pi r_{i}^{3 /\left(n_{i}+1\right)}\left(r_{i} r_{o}\right)^{1 / 2}} \operatorname{Im}\left(F_{1 t h D H}\right),
\end{aligned}
$$

and

$$
\begin{aligned}
& Q_{t N}=\frac{\pi}{2 \mu} \frac{1}{T_{c N}}\left(\frac{\Delta p_{t N}}{h}\right), \\
& Q_{t D H}=\frac{3^{n_{i}+1} \pi^{n_{i}+2} k_{i} r_{M}^{3}\left(r_{i} r_{o}\right)^{\left(n_{i}+1\right) / 2}}{\left(n_{i}+1\right)^{n_{i}+1}\left(n_{i}+4\right) \mu} \frac{1}{\left[\operatorname{Im}\left(F_{l t h D H}\right)\right]^{n_{i}+1}}\left(\frac{\Delta p_{f D H}}{h}\right)^{n_{i}+1}
\end{aligned}
$$

where

$$
\begin{aligned}
& T_{c N}=\frac{\left(r_{i}+r_{o}\right)\left(5 r_{i}^{2}-2 r_{i} r_{o}+5 r_{o}^{2}\right)}{\left(r_{i} r_{o}\right)^{7 / 2}} . \\
& \operatorname{Im}\left(F_{l t h D H}\right)=\operatorname{Im}\left\{F_{l}\left[-\frac{3}{n_{i}+1} ; \frac{1}{2} ; \frac{1}{2} ; \frac{n_{i}-2}{n_{i}+1} ; 1,\left(\frac{r_{i}}{r_{o}}\right)^{ \pm 1}\right]\right\} .
\end{aligned}
$$

Here, $F_{1}[\ldots]$ is the Appell hypergeometric function and $\operatorname{Im}\left(F_{1}\right)$ is the imaginary part of this function.

Taking into account Eqs (3.22) and (3.28) and reusing the principle of superposition (3.6) or (3.9), we may write

$$
\begin{aligned}
& Q_{f}=\frac{4}{3 \mu F_{c N}}\left\{1+\frac{3 \cdot 2^{n_{i}} \pi^{n_{i}+1} k_{i} f_{M}^{2}\left(f_{i} f_{o}\right)^{\left(n_{i}+1\right) / 2} F_{c N}}{\left(n_{i}+1\right)^{n_{i}+1}\left(n_{i}+3\right)\left[\operatorname{Im}\left(F_{1 f h D H}\right)\right]^{n_{i}+1}}\left(\frac{\Delta p}{h}\right)^{n_{i}}\right\}\left(\frac{\Delta p}{h}\right), \\
& Q_{t}=\frac{\pi}{2 \mu T_{c N}}\left\{1+\frac{2 \cdot 3^{n_{i}+1} \pi^{n_{i}+1} k_{i} r_{M}^{3}\left(r_{i} r_{o}\right)^{\left(n_{i}+1\right) / 2} T_{c N}}{\left(n_{i}+1\right)^{n_{i}+1}\left(n_{i}+4\right)\left[\operatorname{Im}\left(F_{1 t h D H}\right)\right]^{n_{i}+1}}\left(\frac{\Delta p}{h}\right)^{n_{i}}\right\}\left(\frac{\Delta p}{h}\right) .
\end{aligned}
$$

The flow velocities through the thin porous layer composed of cosine curve capillaries, modelled as the equivalent matrix of constant cross-section capillaries, will be given, respectively, as

$$
\begin{aligned}
& \mathrm{v}_{f}=\frac{f_{M}^{2} \varphi_{p} \Psi_{1 f c}}{3 \mu}\left\{1+\frac{3 k_{i} f_{M}^{n_{i}} \psi_{2 f c}}{\left(n_{i}+3\right)}\left(-\frac{d p}{d y}\right)^{n_{i}}\right\}\left(-\frac{d p}{d y}\right) \\
& \mathrm{v}_{t}=\frac{r_{M}^{2} \varphi_{p} \psi_{1 t c}}{8 \mu}\left\{1+\frac{4 k_{i} r_{M}^{n_{i}} \psi_{2 t c}}{2^{n_{i}}\left(n_{i}+4\right)}\left(-\frac{d p}{d y}\right)^{n_{i}}\right\}\left(-\frac{d p}{d y}\right)
\end{aligned}
$$

where 


$$
\begin{gathered}
\psi_{1 f c}=\frac{2}{f_{M}^{3} F_{c N}}, \quad \psi_{2 f c}=\frac{2^{n_{i}} \pi^{n_{i}+1} F_{c N}}{\left(n_{i}+1\right)^{n_{i}+1}\left[\operatorname{Im}\left(F_{1 f h D H}\right)\right]^{n_{i}+1}} \times\left\{\begin{array}{l}
\left(\frac{f_{o}^{\left(n_{i}+1\right) / 2}}{f_{M}^{\left(n_{i}-5\right) / 2}}\right) \\
\left(\begin{array}{l}
\left(f_{i}^{\left(n_{i}+1\right) / 2}\right. \\
f_{M}^{\left(n_{i}-5\right) / 2}
\end{array}\right),
\end{array}\right. \\
\psi_{l t c}=\frac{4}{r_{M}^{4} T_{c N}}, \quad \psi_{2 t c}=\frac{2^{n_{i}-1} 3^{n_{i}+1} \pi^{n_{i}+1} T_{c N}}{\left(n_{i}+1\right)^{n_{i}+1}\left[\operatorname{Im}\left(F_{l t h D H}\right)\right]^{n_{i}+1}} \times\left\{\begin{array}{l}
\left(\begin{array}{l}
\left(r_{o}^{\left(n_{i}+1\right) / 2}\right. \\
r_{M}^{\left(n_{i}-7\right) / 2}
\end{array}\right), \\
\left(\begin{array}{l}
r_{i}^{\left(n_{i}+1\right) / 2} \\
r_{M}^{\left(n_{i}-7\right) / 2}
\end{array}\right)
\end{array}\right.
\end{gathered}
$$

and $\psi_{1 f c}, \psi_{l t c}$ are the first hindrance factors, $\psi_{2 f c}, \psi_{2 t c}$ are the second hindrance factors for the cosine curve capillaries.

\section{Conclusions}

In this paper an approximate mathematical method for obtaining the analytical relations between pressure drops and volumetric flow rates in symmetrically corrugated capillary fissures and tubes is presented and applied to study the flow of DeHaven fluids.

Taking into account the considerations on the flows through rectilinear capillaries of constant crosssections, a simple method to describe the flows through convergent-divergent (in general) capillaries with variable cross-sections (rectilinear and curvilinear) was presented.

The presented method is an approximate method because it does not take into account the cross flows which may appear in capillaries of variable cross-sections; these flows can have an essential effect on the pressure drops in the cases of great changes of the cross-sections for very short capillaries.

The method is illustrated by five examples of capillary fissures or tubes with convergent-divergent and divergent-convergent shape, namely: wedge and cone geometries, the parabolic, hyperbolic, hyperbolic cosine and cosine curve.

Each case of the capillary geometry finishes with formulae for the flow velocity through a thin porous layer. To compare the obtained velocities with the flow velocities through porous layer composed by a uniform matrix of rectilinear capillaries hindrance factors have been introduced; the form of these factors indicates that the pressure drops are the same independently of order of convergence and divergence in the capillary.

These factors are always less than one what indicates that the flow velocity through the matrix of corrugated capillaries is also less than the flow velocity through the matrix of rectilinear capillaries.

\section{Appendix}

In this Appendix we present analytical expressions for the integrals appearing in the previous sections of the this paper.

The first of them, for rectilinear capillaries of variable cross-sections, are:

- for the convergent-divergent capillary 


$$
\left.J_{n}\right|_{-h / 2} ^{+h / 2}=\int_{-h / 2}^{+h / 2} \frac{d y}{(a+b|y|)^{n}}=\left.J_{n_{1}}\right|_{-h / 2} ^{0}+\left.J_{n_{2}}\right|_{0} ^{+h / 2}
$$

where

$$
\begin{aligned}
& \left.J_{n_{1}}\right|_{-h / 2} ^{0}=\int_{-h / 2}^{0} \frac{d y}{(a-b y)^{n}}=\left.\frac{1}{(n-1) b} \frac{1}{(a-b y)^{n-1}}\right|_{-h / 2} ^{0}=\frac{1}{(n-1) b}\left[a^{1-n}-\left(a+\frac{b h}{2}\right)^{1-n}\right], \\
& \left.J_{n_{2}}\right|_{0} ^{+h / 2}=\int_{0}^{+h / 2} \frac{d y}{(a+b y)^{n}}=-\left.\frac{1}{(n-1) b} \frac{1}{(a+b y)^{n-1}}\right|_{0} ^{h / 2}=\frac{1}{(n-1) b}\left[a^{1-n}-\left(a+\frac{b h}{2}\right)^{l-n}\right],
\end{aligned}
$$

and

$$
\left.J_{n}\right|_{-h / 2} ^{+h / 2}=\frac{2}{(n-1) b}\left[a^{1-n}-\left(a+\frac{b h}{2}\right)^{1-n}\right]
$$

- for the divergent-convergent capillary

$$
\left.J_{n}\right|_{-h / 2} ^{+h / 2}=\int_{-h / 2}^{+h / 2} \frac{d y}{(a-b|y|)^{n}}=\frac{2}{(n-1) b}\left[\left(a-\frac{b h}{2}\right)^{1-n}-a^{1-n}\right]
$$

here

$$
n=\left\{\begin{array}{ll}
\frac{n_{i}+3}{n_{i}+1} & \text { for a fissure } \\
\frac{n_{i}+4}{n_{i}+1} & \text { for a tube }
\end{array} ; \quad \text { if } \quad n_{i}=0 \quad \text { then } \quad n=\left\{\begin{array}{l}
3 \\
4
\end{array}\right.\right.
$$

The next integrals, for parabolic capillaries, are as follows

for the Newtonian flows we have, respectively

$$
J_{3}=\int \frac{d y}{\left(a \pm b y^{2}\right)^{3}}=\frac{y}{4 a\left(a \pm b y^{2}\right)^{2}}+\frac{3 y}{8 a^{2}\left(a \pm b y^{2}\right)}+\frac{3}{8 a^{2}} I( \pm)
$$

and

$$
\begin{aligned}
& \left.J_{3}\right|_{-h / 2} ^{+h / 2}=\frac{h}{4 a\left(a \pm \frac{b h^{2}}{4}\right)^{2}}+\frac{3 h}{8 a^{2}\left(a \pm \frac{b h^{2}}{4}\right)}+\left.\frac{3}{8 a^{2}} I( \pm)\right|_{-h / 2} ^{+h / 2}, \\
& J_{4}=\int \frac{d y}{\left(a \pm b y^{2}\right)^{4}}=\frac{y}{6 a\left(a \pm b y^{2}\right)^{3}}+\frac{5 y}{24 a^{2}\left(a \pm b y^{2}\right)^{2}}+\frac{5 y}{16 a^{3}\left(a \pm b y^{2}\right)}+\frac{5}{16 a^{3}} I( \pm)
\end{aligned}
$$

and 


$$
\begin{aligned}
& \left.J_{4}\right|_{-h / 2} ^{+h / 2}=\frac{h}{6 a\left(a \pm \frac{b h^{2}}{4}\right)^{3}}+\frac{5 h}{24 a^{2}\left(a \pm \frac{b h^{2}}{4}\right)^{2}}+\frac{5 h}{16 a^{3}\left(a \pm \frac{b h^{2}}{4}\right)}+\left.\frac{5}{16 a^{3}} I( \pm)\right|_{-h / 2} ^{+h / 2} \\
& I( \pm)=\int \frac{d y}{\left(a \pm b y^{2}\right)}=\left\{\begin{array}{l}
\frac{1}{\sqrt{a b}} \arctan \frac{b y}{\sqrt{a b}} \\
\frac{1}{\sqrt{a b}} \operatorname{arctanh} \frac{b y}{\sqrt{a b}}
\end{array},\left.\quad I( \pm)\right|_{-h / 2} ^{+h / 2}=\left\{\begin{array}{l}
\frac{2}{\sqrt{a b}} \arctan \frac{b h}{2 \sqrt{a b}} \\
\frac{2}{\sqrt{a b}} \operatorname{arctanh} \frac{b h}{2 \sqrt{a b}}
\end{array}\right.\right.
\end{aligned}
$$

for the additional flows we have

$$
J_{n}=\int \frac{d y}{\left(a \pm b y^{2}\right)^{n}}=\frac{y}{a^{n}} F\left[\frac{1}{2}, n ; \frac{3}{2} ; \mp \frac{b y^{2}}{a}\right]
$$

where $F[\ldots]$ is a hypergeometric function $[44,45]$ defined by the Gauss series [46]

$$
F[a, b ; c ; z]=\sum_{s=0}^{\infty} \frac{(a)_{s}(b)_{s}}{(c)_{s} s !} z^{s}=1+\frac{a b}{c} z+\frac{a(a+1) b(b+1)}{c(c+1) 2 !} z^{2}+\ldots
$$

here, for convenience, we used the Pochhammer symbol notation for the shifted factorial

$$
(a)_{s}:=\left\{\begin{array}{l}
a(a+1) \ldots(a+s-1) \\
1
\end{array} \text { if } \quad s=\left\{\begin{array}{l}
1,2, \ldots, \\
0 .
\end{array}\right.\right.
$$

Accordingly, we have

$$
(a)_{s}=\frac{\Gamma(a+s)}{\Gamma(a)}
$$

which is used as a definition for the shifted factorial in the case when $s$ is not necessarily a nonnegative integer. Introducing in (A.11) the limits of integration we will obtain

$$
\left.J_{n}\right|_{-h / 2} ^{+h / 2}=\frac{h}{a^{n}} F\left[\frac{1}{2}, n ; \frac{3}{2} ; \mp \frac{b}{a}\left(\frac{h}{2}\right)^{2}\right] .
$$

The third integrals, for hyperbolic capillaries, are given by the following expressions:

for the Newtonian flows we have

$$
J_{3}=\int \frac{d y}{\left(a \pm b y^{2}\right)^{3 / 2}}=\frac{y}{a\left(a \pm b y^{2}\right)^{1 / 2}}
$$


and

$$
\left.J_{3}\right|_{-h / 2} ^{+h / 2}=\frac{h}{a\left(a \pm \frac{b h^{2}}{4}\right)^{1 / 2}}
$$

or

$$
J_{4}=\int \frac{d y}{\left(a \pm b y^{2}\right)^{2}}=\frac{y}{2 a\left(a \pm b y^{2}\right)}+\frac{1}{2 a} I( \pm)
$$

and

$$
\left.J_{4}\right|_{-h / 2} ^{+h / 2}=\frac{h}{2 a\left(a \pm \frac{b h^{2}}{4}\right)}+\left.\frac{1}{2 a} I( \pm)\right|_{-h / 2} ^{+h / 2}
$$

for the additional flows there are

$$
J_{n}=\int \frac{d y}{\left(a \pm b y^{2}\right)^{n / 2}}=\frac{y}{a^{n / 2}} F\left[\frac{1}{2}, \frac{n}{2} ; \frac{3}{2} ; \mp \frac{b y^{2}}{a}\right]
$$

and

$$
\left.J_{n}\right|_{-h / 2} ^{+h / 2}=\frac{h}{a^{n / 2}} F\left[\frac{1}{2}, \frac{n}{2} ; \frac{3}{2} ; \mp \frac{b}{a}\left(\frac{h}{2}\right)^{2}\right]
$$

The fourth and fifth integrals, for hyperbolic cosine capillaries, are as follows:

- the forth ones:

for the Newtonian flows we have

$$
J_{3}=\int \frac{d y}{[a \cosh (b y)]^{3}}=\frac{1}{a^{3} b}\left\{\frac{\sinh (b y)}{2[\cosh (b y)]^{2}}+\frac{1}{2} \arctan [\sinh (b y)]\right\}
$$

and

$$
\left.J_{3}\right|_{-h / 2} ^{+h / 2}=\frac{1}{a^{3} b}\left\{\frac{\sinh \left(\frac{b h}{2}\right)}{\left[\cosh \left(\frac{b h}{2}\right)\right]^{2}}+\arctan \left[\sinh \left(\frac{b h}{2}\right)\right]\right\}
$$

or

$$
J_{4}=\int \frac{d y}{[a \cosh (b y)]^{4}}=\frac{1}{a^{4} b}\left\{1-\frac{[\tanh (b y)]^{2}}{3}\right\} \tanh (b y)
$$

and

$$
\left.J_{4}\right|_{-h / 2} ^{+h / 2}=\frac{2}{a^{4} b}\left\{1-\frac{\left[\tanh \left(\frac{b h}{2}\right)\right]^{2}}{3}\right\} \tanh \left(\frac{b h}{2}\right),
$$


for the additional flows there are

$$
J_{n}=\int \frac{d y}{[a \cosh (b y)]^{n}}=\frac{-i \operatorname{sech}^{n-1}(b y)}{(n-1) a^{n} b} F\left[\frac{1}{2}, \frac{1-n}{2} ; \frac{3-n}{2} ; \cosh ^{2}(b y)\right],
$$

where: $i=\sqrt{-1}$, and the real value of the definite integral $\left.J_{n}\right|_{-h / 2} ^{+h / 2}$ is equal to

$$
\left.J_{n}\right|_{-h / 2} ^{+h / 2}=\frac{2 \operatorname{sech}^{n-1}\left(\frac{b h}{2}\right)}{(n-1) a^{n} b} \operatorname{Im}\left(F\left[\frac{1}{2}, \frac{1-n}{2} ; \frac{3-n}{2} ; \cosh ^{2}\left(\frac{b h}{2}\right)\right]\right)
$$

here $\operatorname{Im}(F[\ldots])$ is the real value of the imaginary part of the hypergeometric function $F[\ldots]$;

- the fifth ones:

for the Newtonian flows there are

$$
J_{3}=\int[a \cosh (b y)]^{3} d y=\frac{3 a^{3}}{4 b}\left[\sinh (b y)+\frac{1}{9} \sinh (3 b y)\right]
$$

and

$$
\left.J_{3}\right|_{-h / 2} ^{+h / 2}=\frac{3 a^{3}}{2 b}\left[\sinh \left(\frac{b h}{2}\right)+\frac{1}{9} \sinh \left(\frac{3 b h}{2}\right)\right]
$$

or

$$
J_{4}=\int[a \cosh (b y)]^{4} d y=\frac{a^{4}}{8 b}\left[3 b y+2 \sinh (2 b y)+\frac{1}{4} \sinh (4 b y)\right]
$$

and

$$
\left.J_{4}\right|_{-h / 2} ^{+h / 2}=\frac{a^{4}}{4 b}\left[\frac{3 b h}{2}+2 \sinh (b h)+\frac{1}{4} \sinh (2 b h)\right]
$$

for the additional flows there are

$$
J_{n}=\int \frac{d y}{[a \cosh (b y)]^{-n}}=\frac{i \cosh ^{n+1}(b y)}{(n-1) a^{-n} b} F\left[\frac{1}{2}, \frac{1+n}{2} ; \frac{3+n}{2} ; \cosh ^{2}(b y)\right],
$$

and the real value of the definite integral $\left.J_{n}\right|_{-h / 2} ^{+h / 2}$ is equal to

$$
\left.J_{n}\right|_{-h / 2} ^{+h / 2}=\frac{2 \cosh ^{n+1}\left(\frac{b h}{2}\right)}{(n+1) a^{-n} b} \operatorname{Im}\left(F\left[\frac{1}{2}, \frac{1+n}{2} ; \frac{3+n}{2} ; \cosh ^{2}\left(\frac{b h}{2}\right)\right]\right) .
$$

The last integrals, for cosine curve capillaries, are given by the following expressions: for the Newtonian flows we have 


$$
\begin{aligned}
& J_{3}=\int \frac{d y}{[a \mp b \cos (k y)]^{3}}=\frac{1}{2 k\left(a^{2}-b^{2}\right)}\left\{\frac{ \pm b \sin (k y)}{[a \mp b \cos (k y)]^{2}}+\right. \\
& \left.+\frac{3 a}{\left(a^{2}-b^{2}\right)[a \mp b \cos (k y)]}+\frac{ \pm b \sin (k y)}{\left(a^{2}-b^{2}\right)} \frac{2}{\sqrt{a^{2}-b^{2}}} \arctan \frac{\sqrt{a^{2}-b^{2}} \tan \left(\frac{k y}{2}\right)}{a \mp b}\right\}
\end{aligned}
$$

and

$$
\begin{aligned}
& \left.J_{3}\right|_{-h / 2} ^{+h / 2}=\frac{1}{k\left(a^{2}-b^{2}\right)}\left\{\frac{ \pm b \sin \left(\frac{k h}{2}\right)}{\left[a \mp b \cos \left(\frac{k h}{2}\right)\right]^{2}}+\frac{3 a}{\left(a^{2}-b^{2}\right)} \frac{ \pm b \sin \left(\frac{k h}{2}\right)}{\left[a \mp b \cos \left(\frac{k h}{2}\right)\right]}+\right. \\
& \left.+\frac{2 a^{2}+b^{2}}{\left(a^{2}-b^{2}\right)} \frac{2}{\sqrt{a^{2}-b^{2}}} \arctan \frac{\sqrt{a^{2}-b^{2}} \tan \left(\frac{k h}{4}\right)}{a \mp b}\right\} ;
\end{aligned}
$$

or

$$
\begin{aligned}
& J_{4}=\int \frac{d y}{[a \mp b \cos (k y)]^{4}}=\frac{1}{3 k\left(a^{2}-b^{2}\right)}\left\{\frac{ \pm b \sin (k y)}{[a \mp b \cos (k y)]^{3}}+\frac{5 a}{\left(a^{2}-b^{2}\right)} \frac{ \pm b \sin (k y)}{[a \mp b \cos (k y)]^{2}}+\right. \\
& \left.+\frac{11 a^{2}+4 b^{2}}{2\left(a^{2}-b^{2}\right)^{2}} \frac{ \pm b \sin (k y)}{[a+b \cos (k y)]}+\frac{6 a^{3}+9 a b^{2}}{\left(a^{2}-b^{2}\right)^{2}} \frac{1}{\sqrt{a^{2}-b^{2}}} \arctan \frac{\sqrt{a^{2}-b^{2}} \tan \left(\frac{k y}{2}\right)}{a \mp b}\right\}
\end{aligned}
$$

and

$$
\begin{aligned}
& \left.J_{4}\right|_{-h / 2} ^{+h / 2}=\frac{2}{3 k\left(a^{2}-b^{2}\right)}\left\{\frac{ \pm b \sin \left(\frac{k h}{2}\right)}{\left[a \mp b \cos \left(\frac{k h}{2}\right)\right]^{3}}+\frac{5 a}{\left(a^{2}-b^{2}\right)} \frac{ \pm b \sin \left(\frac{k h}{2}\right)}{\left[a \mp b \cos \left(\frac{k h}{2}\right)\right]^{2}}+\right. \\
& \left.+\frac{11 a^{2}+4 b^{2}}{2\left(a^{2}-b^{2}\right)^{2}} \frac{ \pm b \sin \left(\frac{k h}{2}\right)}{\left.a \mp b \cos \left(\frac{k h}{2}\right)\right]}+\frac{6 a^{3}+9 a b^{2}}{\left(a^{2}-b^{2}\right)^{2}} \frac{1}{\sqrt{a^{2}-b^{2}}} \arctan \frac{\sqrt{a^{2}-b^{2}} \tan \left(\frac{k h}{4}\right)}{a \mp b}\right\}
\end{aligned}
$$

for the additional flows we have $[44,45]$

$$
J_{n}=\int \frac{d y}{[a \mp b \cos (k y)]^{n}}=\frac{-i}{(n-1) k \sqrt{a^{2}-b^{2}}[a \mp b \cos (k y)]^{n-1}} F_{1}[\ldots]
$$

where $F_{l}[\ldots]$ is the Appell hypergeometric function described here by the formula 


$$
F_{1}[\ldots]=F_{1}\left[1-n ; \frac{1}{2}, \frac{1}{2} ; 2-n ; \frac{a \mp b \cos (k y)}{a \pm b}, \frac{a \mp b \cos (k y)}{a \mp b}\right] .
$$

Generally, the Appell hypergeometric function $F_{l}(x, y)$ is defined by the following double hypergeometric series [46]

$$
F_{1}\left[a ; b_{1}, b_{2} ; c ; x, y\right]=\sum_{m=0}^{\infty} \sum_{n=0}^{\infty} \frac{(a)_{m+n}\left(b_{1}\right)_{m}\left(b_{2}\right)_{n}}{(c)_{m+n} m ! n !} x^{m} y^{n}
$$

It is easy to see that it is a bivariate generalization of the Gauss hypergeometric series defined by formula (A.7). Introducing in (A.20) the limits of integration we will obtain

$$
\left.J_{n}\right|_{-h / 2} ^{+h / 2}=\frac{2}{(n-1) k \sqrt{a^{2}-b^{2}}\left[a \mp b \cos \left(\frac{k h}{2}\right)\right]^{n-1}} \operatorname{Im}\left(F_{1}[\ldots]\right)
$$

where

$$
\operatorname{Im}\left(F_{1}[\ldots]\right)=\operatorname{Im}\left(F_{1}\left[1-n ; \frac{1}{2}, \frac{1}{2} ; 2-n ; \frac{a \mp b \cos \left(\frac{k h}{2}\right)}{a \pm b}, \frac{a \mp b \cos \left(\frac{k h}{2}\right)}{a \mp b}\right]\right)
$$

here, $\operatorname{Im}\left(F_{1}[\ldots]\right)$ is the imaginary part of the Appell hypergeometric function $F_{1}[\ldots]$.

\section{Nomenclature}

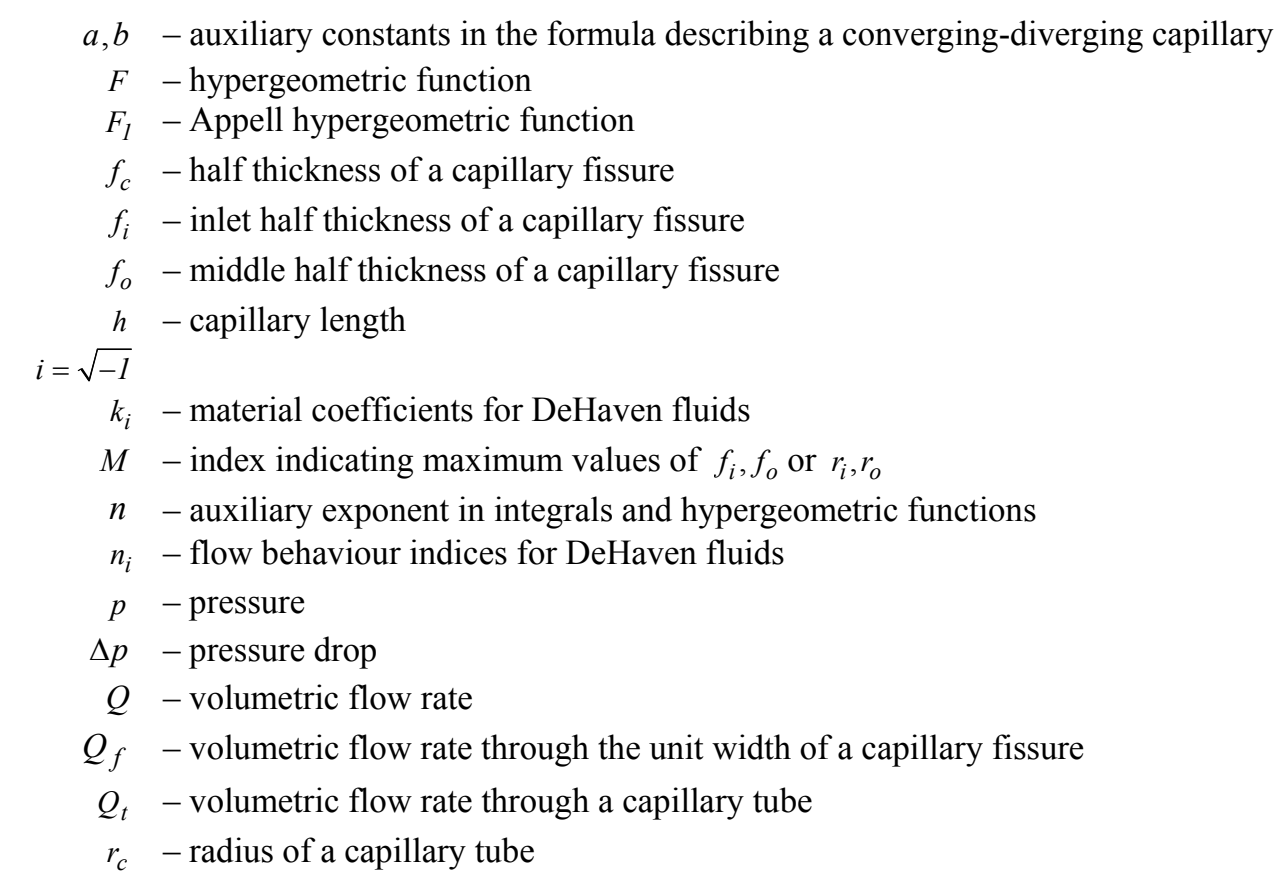


$r_{i}$ - inlet radius of a capillary tube

$r_{o}$ - middle radius of a capillary tube

$v_{f}$ - flow velocity through a thin porous layer modelled by capillary fissures

$v_{t}-$ flow velocity through a thin porous layer modelled by capillary tubes

$\mu \quad$ - fluid viscosity

$\varphi_{p} \quad$ - porosity of a porous layer

$\psi_{1 f}, \psi_{l t}-$ first hindrance factor for a capillary fissure or tube, respectively

$\psi_{2 f,} \psi_{2 t}-$ second hindrance factor for a capillary fissure or tube, respectively

\section{References}

[1] Bear J. (1972): Dynamics of Fluids in Porous Media. - New York: Elsevier.

[2] Greenkorn R.A. (1983): Flow Phenomena in Porous Media. - New York: Marcel Dekker Inc..

[3] Nield D.A., Bejan A. (1995): Convection in Porous Media. - $2^{\text {nd }}$ ed., New York: Springer-Verlag.

[4] Vafai K. (1984): Convective flow and heat transfer in variable porosity media. - J. Fluid Mech., vol.147, pp.233-259.

[5] Vafai K. (1986): Analysis of the channelling effect in variable porosity media. - ASME J. Energy Res. Technol., vol.108, 131-139.

[6] Vafai K. (2000): Handbook of Porous Media. - $1^{\text {st }}$ ed., New York: Marcel Dekker Inc..

[7] Vafai K. (2005): Handbook of Porous Media. $-2^{\text {nd }}$ ed., New York: Taylor \& Francis Group.

[8] Hadim H. and Vafai K. (2000): Overview of current computational studies of heat transfer in porous media and their applications: forced convection and multiphase transport. - In: Advances in Numerical Heat Transfer. New York: Taylor \& Francis Group.

[9] Vafai K. and Hadim H. (2000): Overview of current computational studies of heat transfer in porous media and their applications: natural convection and mixed convection. - In: Advances in Numerical Heat Transfer. - New York: Taylor \& Francis Group.

[10] Vafai K. and Tien C.L. (1982): Boundary and inertia effects on convective mass transfer in porous media. - Int. J. Heat Mass Transfer, vol.25, pp.1183-1190.

[11] Amiri A. and Vafai K. (1998): Transient analysis of incompressible flow through a packed bed. - Int. J. Heat Mass Transfer, vol.41, pp.4259-4279.

[12] Alazmi B. and Vafai K. (2002): Constant wall heat flux boundary conditions in porous media under local thermal non-equilibrium conditions. - Int. J. Heat Mass Transfer, vol.45, pp.3071-3087.

[13] Khanafer K.M., Bull J.L., Pop I. and Berguer R. (2007): Influence of pulsatile blood flow and heating scheme on the temperature distribution during hyperthermia treatment. - Int. J. Heat Mass Transfer, vol.50, pp.4883-4890.

[14] Peng X.F. and Wu H.L. (2005): Pore- scale transport phenomena in porous media. - In: Transport Phenomena in Porous Media III, Elsevier, New York, pp.366-398.

[15] Harris S.D., Fishert Q.J, Karimi-Fard M., Vaszi A.Z. and Wu K. (2005): Modelling the effects of faults and fractures on fluid flow in petroleum reservoirs. - In: Transport Phenomena in Porous Media III, Elsevier, New York, pp.441-476.

[16] Karamouz M., Ahmadi A. and Akhbari M. (2001): Groundwater Hydrology: Engineering, Planning, and Management. - CRC Press, New York: Taylor \& Francis Group.

[17] Yeh W.W. (2015): Optimization methods for groundwater modelling and management. - Hydrogeology Journal, vol.23, pp.1051-1065.

[18] Jivkov A.P., Hollis C., Etiese F., McDonald S.A. and Withers P.J. (2013): A novel architecture for pore network modelling with applications to permeability of porous media. - J. Hydrology, vol.486, pp.246-258. 
[19] Xiong Q., Baychev T.G. and Jivkov A.P. (2016): Review of pore network modelling of porous media: experimental characterisations, network constructions and applications to reactive transport. - J. Contaminant Hydrology, vol.192, pp.101-117.

[20] Mazaheri A.R., Zerai B., Ahmadi G., Kadambi J.R., Saylor B.Z., Oliver M. and Smith D.H. (2005): Computer simulation of the flow through a lattice flow-cell model. - Advances in Water Resources, vol.28, pp.1267-1279.

[21] Joekar-Niasar V., Hassanizadeh S.M., Pyrak-Nolte L.J. and Berentsen C. (2009): Simulating drainage and imbibition experiment in a high-porosity micromodel using an unstructured pore network model. - Water Resource Research, vol.45, No.2, DOI: 10.1029/2007WR006641.

[22] Nsir K. and Schäfer G. (2010): A pore-throat model based on grain-size distribution to quantify gravity-dominated DNAPL instabilities in a water saturated homogeneous porous medium. - Comptes Rendus Geoscience, vol.342, pp.881-891.

[23] Held R.J. and Celia M.A (2001): Modeling support of functional relationships between capillary pressure, saturation, interfacial area and common lines. - Adv. Water Res., vol.24, pp.325-343.

[24] Hilpert M., Miller C.T. and Gray W.G. (2003): Stability of a fluid-fluid interface in a biconical pore segment. - J. Call. Interface Sci., vol.267, pp.397-407.

[25] Acharya R.C., van der Zee S.E.A.T.M. and Leijense A. (2004): Porosity-permeability properties generated with a new 2-parameter $3 D$ hydraulic pore-network model for consolidated and unconsolidated porous media. - Adv. Water Res., vol.27, pp.707-723.

[26] Walicki E., Walicka A. Michalski D. and Ratajczak P. (1998): Approximate analysis for conical flow of generalized second grade fluid. - Les Cahiers de Rhéologie, vol.16, No.1, pp.309-316.

[27] Walicki E. and Walicka A. (2000): Pressure drops in a wedge flow of generalized second grade fluids of a powerlaw type and a Bingham type. - Les Cahiers de Rhéologie, vol.17, No.1, pp.541-550.

[28] Walicki E. and Walicka A. (2000): Conical flow of generalized second grade fluids. - Chem. Proc. Eng., vol.21, No.1, pp.75-85.

[29] Walicki E. and Walicka A. (2002): Convergent flows of molten polymers modeled by generalized second-grade fluids of a power type. - Mech. Comp. Mat., vol.38, No.1, pp.89-94.

[30] Walicka A. and Walicki E. (2010): Pressure drops in convergent flows of polymer melts. - Int. J. Appl. Mech. Eng., vol.15, No.4, pp.1273-1285.

[31] Sochi T. (2010): The flow of Newtonian fluids in axisymmetric corrugated tubes. - arXiv:1006.1515v1.

[32] Sochi T. (2011): Newtonian flow in converging-diverging capillares. - arXiv:1108.0163v1.

[33] Sochi T. (2011): The flow of power-law fluids in axisymmetric corrugated tubes. - J. Petrol. Sci. Eng., vol.78, pp.582-585.

[34] Sochi T. (2015): Navier-Stokes flow in converging-diverging distensible tubes. - Alexandria Eng. J., vol.54, pp.713-723.

[35] Walicka A. (2018): Flows of Newtonian and power-law fluids in symmetrically corrugated capillary fissures and tubes. - Int. J. Appl. Mech. Eng., vol.23, No.1, pp.187-211.

[36] Walicka A. (2018): Simulation of the flow through porous layers composed of converging-diverging capillary fissures or tubes. - Int. J. Appl. Mech. Eng., vol.23, No.1, pp.161-185.

[37] Vossoughi S. (1999): Flow of non-Newtonian fluids in porous media. - In: Advances in the Flow and Rheology of NonNewtonian Fluids, Elsevier, New York, pp.1183-1235.

[38] Pearson J.R.A. and Tardy P.M.J. (2002): Models for flow of non-Newtonian and complex fluids through porous media. - J. Non-Newt. Fluid Mech., vol.102, pp.447-473.

[39] Perrin C.L., Tardy P.M.J., Sorbie K.S. and Crawshaw J.C. (2006): Experimental and modelling study of Newtonian and non-Newtonian fluid flow in pore network micromodels. - J. Colloid. Interface Sci., vol.295, pp.542-550. 
[40] Vossoughi S. and Al-Husaini O.S. (1994): Rheological characterisation of the coal/oil/water slurries and the effect of polymer. $-19^{\text {th }}$ international Technical Conference on Coal Utilization \& Fuel Systems, Clearwater, Florida, USA, pp.115-122.

[41] DeHaven E.S (1953): Control valve design for viscous pseudoplastic fluids. - JEC Equipment and Design, vol.51, No.7, pp.63A-66A.

[42] DeHaven E.S (1959): Extruder design for pseudoplastic fluids. - Ind. Eng. Chem., vol.51, No.7, pp.813-816.

[43] Walicka A. (2017): Rheology of Fluids in Mechanical Engineering. - Zielona Gora: University Press.

[44] Gradshteyn I.S and Ryzhik I.M. (2014): Tables of Integrals, Series and Products, $8^{\text {th }}$ ed. - Orlando, Florida: Academic Press.

[45] Wolfram: Mathematica 10. - Wolfram Res. Ing.

[46] Olver F.W.J., Lozier D.W., Boisvert R.F. and Clark C.W. (2010): NIST Handbook of Mathematical Functions. NIST \& Cambridge University Press.

Received: January 4, 2018

Revised: March 1, 2018 